\title{
Understanding initial data for black hole collisions
}

\author{
Carlos O. Lousto* and Richard H. Price \\ Department of Physics, University of Utah, Salt Lake City, UT 84112
}

\begin{abstract}
Numerical relativity, applied to collisions of black holes, starts with initial data for black holes already in each other's strong field. For the initial data to be astrophysically meaningful, it must approximately represent conditions that evolved from holes originally at large separation. The initial hypersurface data typically used for computation is based on mathematically simplifying prescriptions, such as conformal flatness of the 3-geometry and longitudinality of the extrinsic curvature. In the case of head on collisions of equal mass holes, there is evidence that such prescriptions work reasonably well, but it is not clear why, or whether this success is more generally valid. Here we study these questions by considering the "particle limit" for head on collisions of nonspinning holes, i.e., the limit of an extreme ratio of hole masses. The mass of the small hole is considered to be a perturbation of the Schwarzschild spacetime of the larger hole, Einstein's equations are linearized in this perturbation, and described by a single gauge invariant spacetime function $\psi$, for each multipole. The resulting quadrupole equations have been solved by numerical evolution for collisions starting from various initial separations, and the evolution is studied on a sequence of hypersurfaces. In particular, we extract hypersurface data, that is $\psi$ and its time derivative, on surfaces of constant background Schwarzschild time. These evolved data can then be compared with "prescribed" data, evolved data can be replaced by prescribed data on any hypersurface, and evolved further forward in time, a gauge invariant measure of deviation from conformal flatness can be evaluated, and other comparisons can be made. The main findings of this study are: (i) For holes of unequal mass the use of prescribed data on late hypersurfaces is not successful. (ii) The failure is likely due to the inability of the prescribed data to represent the near field of the smaller hole. (iii) The discrepancy in the extrinsic curvature is more important than in the 3-geometry. (iv) The use of the more general conformally flat longitudinal data does not notably improve this picture.
\end{abstract}

\section{INTRODUCTION AND BACKGROUND}

The collision of black holes is of great interest as both astrophysics and as a strong-field gravitational interaction with no Newtonian analog. Black hole collisions also may provide the strongest, most observable, source of gravitational radiation that can be detected by gravitational wave observatories now under construction [1]. The strong field nature of the process means that the nonlinear character of Einstein's theory plays a crucial role, so that estimates based on linearized theory are unreliable. For the last stage of coalescence of two rotating holes in a decaying orbit, we have only dimensional estimates. The timeliness of the problem has given rise to an effort to attack the problem by solving Einstein's equations numerically on supercomputers [2].

The numerical solution starts with some "initial value" data, a solution of a subset of Einstein's equations on an initial hypersurface, and then evolves this solution forward in time. The specification of the initial data is tantamount to specifying what problem it is that the computer will be solving, so the initial data must encode the physical parameters (mass, spin, location, momentum) of the colliding objects. These physical parameters are meaningful and well understood for isolated holes, but become increasingly ambiguous as the initial separation between the holes decreases, and the field of each hole has a strong effect on the spacetime geometry of the other. Ultimately, at sufficiently small initial separation, it becomes impossible to make any clear statements about the physical parameters of an individual hole.

In principle, one would like to avoid this difficulty by specifying initial data on a hypersurface in the distant past when the holes were separated by a distance many times the horizon size. When the initial influence of each hole on the other becomes small, the uncertainties in the choice of initial data become negligible. Unfortunately, it is not possible to start the numerical evolution a long time before the collision. Einstein's equations are a nonlinear hyperbolic system, and for such systems instabilities in numerical evolution seem to be a generic feature. While it can be expected that some progress will be made in improving the codes and suppressing the instabilities, it is not

*Electronic Address: lousto@mail.physics.utah.edu 
likely that in the near future these codes will be able to evolve initial data for more than a few dynamical times, the characteristic time scale for black hole processes (around $10^{-5} \mathrm{sec}$ for a solar mass hole). This limitation precludes starting the dynamics with the holes at large distances and means that the initial data must be given on a hypersurface on which the hole-hole interactions are already strong. If the resulting numerical evolutions are to be astrophysically meaningful, it is crucial to have a way of connecting data on this "late" hypersurface to an astrophysical precursor configuration with the holes interacting weakly. In other words, to understand the meaning of initial data on a "late" hypersurface, we must know where that data came from. One approach to providing this connection is to use approximations such as higher-order post-Newtonian methods in order to understand the evolution of the system from an early stage to a stage at which the holes are interacting with intermediate strength. Such an approach may turn out to be sufficient if the numerical evolutions can be stabilized for a relatively long time.

There is reason to hope that it might be possible to give initial data at late times, with relatively little difficulty. The reason is based on the success of a "two phase" approximation method used by Abrahams and Cook [3] and further investigated by Baker and Li [ []. These studies dealt with the head on (zero angular momentum) collisions of two equal-mass nonrotating holes. Data were specified on a very late hypersurface, simply by using a "standard" initial value solution formally representing equal mass holes moving towards each other. This standard solution contained a parameter $P$ which, in the case of an isolated hole, agreed with the momentum of the hole. On the very late hypersurface the separation and momentum were set at the values dictated by Newtonian gravity theory if the holes had started infall from some large initial separation. The standard initial value data was evolved forward in time (with the use of an approximation method). The radiated energy found by this approximation could be compared with the "correct" radiated energy, since numerical relativity results were available for such collisions. The approximation method agreed with numerical relativity to rather good accuracy. This is somewhat a surprise since the "standard" numerical relativity initial data should be very different from the "correct" evolved data. The implication of this agreement is that results for gravitational radiation might not be sensitive to all details of initial data.

We explore this question in the present paper by comparing prescriptions for imposing data on hypersurfaces with the "correct" data evolved from an earlier configuration. In order to do this, of course, we need the ability to evolve a solution for a relatively long time, something that cannot yet be done with the nonlinear Einstein equations. We choose therefore to use the particle approximation, the approximation in which the mass $m$ one of our holes, is much smaller than the mass of the other $M$. We then treat the low-mass hole as a particle of negligible size, and we treat $m / M$ as an expansion parameter. By doing first-order perturbation theory with this parameter, our evolution equations become linear, so that a stable numerical scheme is straightforward to develop.

In an earlier paper [5] (Paper I), we developed the basic mathematics of the particle limit approximation, and of evolving data that was initially stationary. In that paper, Laplace transform methods were used. The method for the present results is a direct numerical solution of the partial differential equations in radius and time (after multipole decomposition). We have compared results of the two completely different methods, and found agreement within the estimated numerical accuracy, thereby adding confidence that there are no mistakes in our numerical methods.

The paper is organized as follows. In Sec. II we give the mathematical formulation of the problem. We base this formulation on the gauge-invariant Moncrief [6] approach to perturbations, so that the variable $\psi$ we use is invariant with respect to perturbative changes in slicing. We need only specify the slicing to zero order in the perturbation, and to that order we take our hypersurfaces to be slices of constant Schwarzschild time. In Sec. II we discuss in detail the freedom in initial data and the standard choices made for reducing that freedom. We go on to explain the particular physically-motivated choice that we make for arriving at a definitive prescription of initial data. In Sec. III, we start by explaining our numerical method for evolving $\psi$. The scheme for solving the linear hyperbolic equation obeyed by $\psi$ is straightforward. The only issue that deserves attention is the source term in the equation for $\psi$. In the particle limit, the small hole is treated as a point particle, and this particle is associated with a stress energy source that enters the $\psi$ equation as the derivative of a delta function of radial position. We describe in Sec. III how the singular source is handled. Numerical results are presented and compared in Sec. III.B. In Sec. IV we discuss the results and their meaning.

\section{MATHEMATICAL FORMULATION}

\section{A. Moncrief-Zerilli formalism}

We will describe the perturbations to the Schwarzschild background, due to the particle, in the notation of Regge

and Wheeler [7] (hereafter RW). The symmetry of the straightline infall of the particle means that there will be no odd parity perturbations. For the even parity perturbations the general form of the line element, with perturbations of a specific multipole index $\ell$, is 


$$
\begin{array}{r}
d s^{2}=d s_{0}^{2}+(1-2 M / r)\left(H_{0}^{\ell} Y_{\ell 0}\right) d t^{2}+(1-2 M / r)^{-1}\left(H_{2}^{\ell} Y_{\ell 0}\right) d r^{2} \\
+r^{2}\left(K^{\ell} Y_{\ell 0}+G^{\ell} \partial^{2} Y_{\ell 0} / \partial \theta^{2}\right) d \theta^{2}+r^{2}\left(\sin ^{2} \theta K^{\ell} Y_{\ell 0}+G^{\ell} \sin \theta \cos \theta \partial Y_{\ell 0} / \partial \theta\right) d \phi^{2} \\
+2 H_{1}^{\ell} Y_{\ell 0} d t d r+2 h_{0}^{\ell}\left(\partial Y_{\ell 0} / \partial \theta\right) d t d \theta+2 h_{1}^{\ell}\left(\partial Y_{\ell 0} / \partial \theta\right) d r d \theta .
\end{array}
$$

Here $d s_{0}^{2}$ is the unperturbed line element for a Schwarzschild spacetime of mass $M$, where $H_{0}^{\ell}, H_{1}^{\ell}, H_{2}^{\ell}, h_{0}^{\ell}, h_{1}^{\ell}, K^{\ell}$ and $G^{\ell}$ are functions of $r, t$, and $Y_{\ell 0}(\theta)$ are the $m=0$ spherical harmonics. For simplicity, from here on we shall drop the $\ell$ index on perturbation functions.

There are two closely related formalisms available for describing the evolution of the perturbations in terms of a single wave function and a single wave equation. The method due to Moncrief [6] uses only information $\left(H_{2}, h_{1}, K, G\right)$ about the 3-geometry of a $t=$ constant hypersurface, and is gauge invariant, i.e., independent of the choice of lapse and shift, and of diffeomorphisms on the hypersurface. The second method, due to Zerilli [8], relies on a specific gauge, the gauge choice introduced by Regge and Wheeler [7], in which $h_{0}, h_{1}$, and $G$ are set to zero.

As in Paper I we shall use the Moncrief formalism. The Moncrief wave function $\psi$, in terms of the perturbations in the RW notation, is

$$
\psi(r, t)=\frac{r}{\lambda+1}\left[K+\frac{r-2 M}{\lambda r+3 M}\left\{H_{2}-r \partial K / \partial r\right\}\right]+2(1-2 M / r)\left(r^{2} \partial G / \partial r-2 h_{1}\right),
$$

where we have used Zerilli's normalization for $\psi$ and his notation

$$
\lambda \equiv(\ell+2)(\ell-1) / 2 .
$$

For simplicity, we shall occasionally present some equations restricted to the RW gauge. Since the Moncrief wave function is gauge invariant, this gauge restriction allows simplicity of presentation, with no other consequences. In this gauge, we omit the $G$ and $h_{1}$ terms in Eq. (2.2).

The basic wave equation for an infalling particle is given in Paper I as

$$
-\frac{\partial^{2} \psi}{\partial t^{2}}+\frac{\partial^{2} \psi}{\partial r *^{2}}-V_{\ell}(r) \psi=\mathcal{S}_{\ell}(r, t) .
$$

Here $r^{*} \equiv r+2 M \ln (r / 2 M-1)$ is the Regge-Wheeler [7] "tortoise" coordinate and $V_{\ell}$ is the Zerilli potential (given, e.g., in Paper I). For a point particle of proper mass $m_{0}$, the stress energy is given by

$$
T^{\mu \nu}=\left(m_{0} / U^{0}\right) U^{\mu} U^{\nu} \delta\left(r-r_{p}[t]\right) \delta^{2}(\Omega) / r^{2},
$$

where $U^{\mu}$ is the particle 4 -velocity. The two dimensional delta function $\delta^{2}(\Omega)$ gives the angular location of the particle trajectory

$$
\delta^{2}(\Omega)=\sum_{\ell, m} Y_{\ell m}(\theta, \phi) Y_{\ell m}^{*}(\theta, \phi)=\sum_{\ell} Y_{\ell 0}(\theta) \sqrt{(2 \ell+1) / 4 \pi}
$$

with the last expression applying for infall along the positive $z$ axis. The time dependent location of the particle $r_{p}(t)$ follows from the geodesic equation and, for a particle starting from rest at $t=0, r=r_{0}$, is the inverse of

$$
\begin{aligned}
T\left(r_{p}\right)= & \sqrt{1-\frac{2 M}{r_{0}}}\left(\frac{r_{0}}{2 M}\right)\left(\frac{r_{p}}{2 M}\right)^{1 / 2} \sqrt{1-\frac{r_{p}}{r_{0}}} \\
& +\left(1+\frac{4 M}{r_{0}}\right)\left(\frac{r_{0}}{2 M}\right)^{3 / 2} \sqrt{1-\frac{2 M}{r_{0}}} \arctan \left[\sqrt{\frac{r_{0}}{r_{p}}-1}\right] \\
& \ln \left[\frac{1-\left(1-4 M / r_{0}\right)\left(r_{p} / 2 M\right)+2 \sqrt{1-2 M / r_{0}} \sqrt{r_{p} / 2 M} \sqrt{1-r_{p} / r_{0}}}{\left(r_{p} / 2 M\right)-1}\right] .
\end{aligned}
$$

From the particle stress energy in (2.5), the source term $\mathcal{S}_{\ell}(r, t)$ on the right hand side of (2.4), is given in Paper I as

$$
\begin{aligned}
\mathcal{S}_{\ell}(r, t) & =-\frac{2(1-2 M / r) \kappa}{r(\lambda+1)(\lambda r+3 M)}\left[-r^{2}(1-2 M / r) \frac{1}{2 U^{0}} \delta^{\prime}\left(r-r_{p}[t]\right)\right. \\
& \left.+\left\{\frac{r(\lambda+1)-M}{2 U^{0}}-\frac{3 M U^{0} r(1-2 M / r)^{2}}{\lambda r+3 M}\right\} \delta\left(r-r_{p}[t]\right)\right]
\end{aligned}
$$


where

$$
\kappa \equiv 8 \pi m_{0} \sqrt{(2 \ell+1) / 4 \pi} .
$$

The total radiated energy after a given $t_{0}$ is

$$
\text { Energy }=\frac{1}{64 \pi} \frac{(\ell+2) !}{(\ell-2) !} \int_{t_{0}}^{\infty}(\dot{\psi})^{2} d t .
$$

\section{B. Hypersurface data for $\psi$}

We choose our data, on a hypersurface of constant $t$, to correspond to the choice usually made for work in numerical relativity. The data, for both the initial 3-geometry and extrinsic curvature, are based on the work of York [9] and collaborators. This approach takes the 3 -geometry of the initial hypersurface to be given by $d \sigma^{2}=\Phi^{4} d \sigma_{\text {flat }}^{2}$. The conformal factor $\Phi$ must, in vacuum, satisfy the equation

$$
\nabla^{2} \Phi=-\frac{1}{8} \Phi^{5} K_{i j} K^{i j}
$$

where $\nabla^{2}$ is the Laplacian taken with respect to the flat background, and where $K_{i j}$ is the extrinsic curvature.

For a constant $t$ slice of the unperturbed Schwarzschild spacetime, the extrinsic curvature vanishes. It follows that $K_{i j}$ is perturbative, and hence the right hand side of (2.11) is a higher order perturbation, which, in our first order perturbation calculation, we can ignore. We denote coordinates in the conformally related flat space with bars, such as $\bar{r}, \bar{z}$, and we note that $\Phi$ must be a harmonic function of these coordinates. We make two different choices for $\Phi$. The first is the solution discussed by Brill and Lindquist [10], and which we will call a BL type solution. This solution follows if we treat $2 \Phi$ as if it were the Newtonian potential of two points, one of mass $M$, and one of mass $m_{p}$. If the first mass is located at the origin of the flat coordinates, and the second is at (flat) coordinate position $\bar{z}=\bar{z}_{p}$, then the BL solution takes the form

$$
\Phi_{\mathrm{BL}}=1+\frac{M}{2 \bar{r}}+\frac{m_{p} / 2}{\left|\bar{r} \mathbf{e}_{r}-\bar{z}_{p} \mathbf{e}_{z}\right|} .
$$

Though the origin and the point $\bar{r} \mathbf{e}_{r}=\bar{z}_{p} \mathbf{e}_{z}$ are coordinate singularities, they are not geometric singularities. Near these coordinate points the divergence of the conformal factor means that the "points" are, in fact, asymptotically flat regions, so the geometry given by (2.12) is actually that of three asymptotically flat spaces connected by two throats. For our purposes here, we take the "particle limit," the limit that $m_{p} \ll M$.

The BL solution is the only choice that was considered in Paper I. A second initial conformal factor that can be considered is that which is "reflection symmetric," in the sense that there are only two asymptotically flat regions, and they are isometric to each other. The method of constructing such solutions was given by Misner [11], and requires that "image" points be used in the conformal space, in such a way that the geometry is symmetric with respect to inversion through a sphere about each of the point singularities. Here we make the solution symmetric only for a sphere about the origin. The singularity at $\bar{r} \mathbf{e}_{r}=\bar{z}_{p} \mathbf{e}_{z}$, in the limit, will be taken to have a zero size inversion sphere around it (the particle limit) and the images inside it will be meaningless. (Alternatively one can construct a solution reflection symmetric for two spheres, and subsequently take the limit; the result is the same.) The conformal factor, for this "Misner" case, is

$$
\Phi_{\mathrm{Mis}}=1+\frac{M}{2 \bar{r}}+\frac{m_{p} / 2}{\left|\bar{r} \mathbf{e}_{r}-\bar{z}_{p} \mathbf{e}_{z}\right|}+\frac{m_{\text {image }} / 2}{\left|\bar{r} \mathbf{e}_{r}-\bar{z}_{\text {image }} \mathbf{e}_{z}\right|} .
$$

It is straightforward to verify that this Misner solution is symmetric for inversion about $\bar{r}=M / 2$ if the parameters $m_{\text {image }}, \bar{z}_{\text {image }}$ are chosen to be $m_{\text {image }}=m_{p} M /\left(2 \bar{z}_{p}\right)$ and $\bar{z}_{\text {image }}=(M / 2)^{2} / \bar{z}_{p}$.

For either the BL form or the Misner form the line element for the 3-geometry can now be written

$$
d \sigma^{2}=\left[1+M / 2 \bar{r}+\sum_{\ell=0,1,2, \cdots} \alpha_{\ell}(\bar{r}) P_{\ell}(\cos \theta)\right]^{4}\left(d \bar{r}^{2}+\bar{r}^{2} d \Omega^{2}\right)
$$


with $d \Omega^{2}=d \theta^{2}+\sin ^{2} \theta d \phi^{2}$. The requirement that $\Phi$ be harmonic means that $\alpha_{\ell}$ must have the form $a_{\ell} \bar{r}^{\ell}+b_{\ell} \bar{r}^{-(\ell+1)}$. We must now put the metric into a form for comparison with the Schwarzschild metric. To do this we introduce a Schwarzschild-like radial coordinate $r$ related to the flat space coordinate $\bar{r}$ by

$$
\bar{r}=(\sqrt{r}+\sqrt{r-2 M})^{2} / 4, \quad r=\bar{r}(1+M / 2 \bar{r})^{2} .
$$

This is only one of many possibilities for such a transformation, but it is the simplest for computation, and has been very convenient for perturbation analysis [12]. This transformation puts (2.14) into the form

$$
d \sigma^{2}=\left[1+\frac{2 m_{p} / \bar{r}}{1+M /(2 \bar{r})} \sum_{\ell=0,1,2 \ldots} \mathcal{F}_{\ell}(\bar{r}) P_{\ell}(\cos \theta)\right]\left(\frac{d r^{2}}{1-2 M / r}+r^{2} d \Omega^{2}\right),
$$

where we have kept only terms first order in $m_{p}$, and, as in Paper I, have introduced the notation $\mathcal{F}_{\ell}(\bar{r})=\left(2 \bar{r} / m_{p}\right) \alpha_{\ell}(\bar{r})$.

The perturbations, in the RW notation, can now be written

$$
K=H_{2}=\frac{2 m_{p} / \bar{r}}{1+M / 2 \bar{r}} \mathcal{F}_{\ell}(\bar{r}) \sqrt{\frac{4 \pi}{2 \ell+1}},
$$

with $h_{1}=G=0$. These perturbations of the initial hypersurface turn out to be in the RW gauge, so we can compare them with the hamiltonian constraint, in the RW gauge, as given by Zerilli [13], and in Paper I, for a particle of proper mass $m_{0}$.

$$
\begin{array}{r}
\left(1-\frac{2 M}{r}\right) \frac{\partial^{2} K}{\partial r^{2}}+\left(3-\frac{5 M}{r}\right) \frac{1}{r} \frac{\partial K}{\partial r}-\frac{\ell(\ell+1)}{2 r^{2}}\left(K+H_{2}\right)-\frac{1}{r^{2}}\left(H_{2}-K\right) \\
-\left(1-\frac{2 M}{r}\right) \frac{1}{r} \frac{\partial H_{2}}{\partial r}=-8 \pi m_{0} \sqrt{\frac{2 \ell+1}{4 \pi}} U^{0}\left(1-\frac{2 M}{r}\right) \frac{1}{r^{2}} \delta\left(r-r_{p}\right),
\end{array}
$$

in which the right hand side comes directly from the stress energy expression (2.5).

For the choice of a conformally flat 3-geometry we have $H_{2}=K$ and Eq. (2.18) becomes a differential equation for $K$. A particular solution of this equation, well behaved at infinity, is

$$
K^{\text {partic }}=\sqrt{\frac{4 \pi}{2 \ell+1}}\left(\frac{2 m_{p} / \bar{r}}{1+M / 2 \bar{r}}\right)\left\{\begin{array}{ll}
\left(\bar{z}_{p} / \bar{r}\right)^{\ell} & \bar{r}>\bar{z}_{p} \\
\left(\bar{r} / \bar{z}_{p}\right)^{(\ell+1)} & \bar{r}<\bar{z}_{p}
\end{array} .\right.
$$

This solution, in fact, corresponds precisely the BL solution of Eq. 2.12). For $\bar{z}_{\text {image }}<M / 2$ and $\bar{r}>M / 2$, a homogeneous solution well behaved at infinity is

$$
K^{\text {homog }}=\sqrt{\frac{4 \pi}{2 \ell+1}}\left(\frac{2 m_{\text {image }} / \bar{r}}{1+M / 2 \bar{r}}\right)\left(\frac{\bar{z}_{\text {image }}}{\bar{r}}\right)^{\ell} .
$$

If we take $\bar{z}_{\text {image }}=(M / 2)^{2} / \bar{z}_{p}$ and $m_{\text {image }}=m_{p} M /\left(2 \bar{z}_{p}\right)$, then the homogeneous solution can be considered to be the solution due to an "image" mass, inside the horizon at $\bar{r}=\bar{z}_{\text {image }}$, and it is straightforward to verify that with these choices of $\bar{z}_{\text {image }}$ and $m_{\text {image }}$ the Misner solution is $K^{\mathrm{Mis}}=K^{\text {partic }}+K^{\text {homog }}$. There are, of course, other possibilities. We could, for instance, consider, with the same choices of $\bar{z}_{\text {image }}$ and $m_{\text {image }}$, the combination $K^{\text {anti }}=K^{\text {partic }}-K^{\text {homog }}$. For this solution $K$ and $\psi$ would vanish on the horizon. This property turns out to be preserved by evolution as is made clear in the discussion below.

By using (2.15) and integrating across the singularity in (2.18) at $r=r_{p}=\bar{z}_{p}\left[1+M /\left(2 \bar{z}_{p}\right)\right]^{2}$, we find that

$$
\begin{aligned}
\Delta K,_{r} & \equiv d K /\left.d r\right|_{r=r_{p}^{+}}-d K /\left.d r\right|_{r=r_{p}^{-}} \\
= & -8 \pi m_{p} \frac{\sqrt{(2 \ell+1) / 4 \pi}}{\bar{z}_{p}^{1 / 2} r_{p}^{3 / 2} \sqrt{1-2 M / r_{p}}} \\
& =-8 \pi m_{0} \frac{\sqrt{(2 \ell+1) / 4 \pi}}{r_{p}^{2}} U^{0} .
\end{aligned}
$$

The 4-velocity component $U^{0}$ is given by 


$$
U^{0}=\frac{\sqrt{1-2 M / r_{0}}}{1-2 M / r_{p}}
$$

so it follows from (2.21) that

$$
m_{p}=m_{0} \sqrt{\left(\frac{\bar{z}_{p}}{r_{p}}\right) \frac{1-2 M / r_{0}}{1-2 M / r_{p}}}=\frac{1}{2} m_{0}\left(1+\sqrt{1-\frac{2 M}{r_{p}}}\right) \sqrt{\frac{1-2 M / r_{0}}{1-2 M / r_{p}}} .
$$

Note that the relationship of the mass parameter $m_{p}$ and the particle mass $m_{0}$ is the same for the Misner case as for the BL case, and hence the same as in Paper I. This must be true, of course, since the symmetrizing image term addition in (2.20) has no discontinuity at $r=r_{p}$.

The initial hypersurface, on which the particle and the hypersurface data are stationary, is always denoted by $t=0$. On hypersurfaces with $t>0$ we shall limit our choice of prescribed data to conformally flat 3 -geometries. The choice of conformal flatness is not, however, preserved by evolution. This means that the 3 -geometry on constant $t$ hypersurfaces will in general not be conformally flat. On such a hypersurface, we will have numerical values only for a single function, the Moncrief function $\psi$. It turns out that we can test $\psi$ for underlying conformal flatness. The combination of metric perturbations

$$
I_{\mathrm{conf}} \equiv H_{2}-K+\frac{2}{r}\left(1-\frac{3 M}{r}\right)\left(h_{1}-\frac{r^{2}}{2} \partial_{r} G\right)-2\left(1-\frac{2 M}{r}\right) \partial_{r}\left(h_{1}-\frac{r^{2}}{2} \partial_{r} G\right) .
$$

is gauge invariant, and clearly vanishes for a 3 -geometry that is in conformally flat form, with $h_{1}=0, G=0$ and $H_{2}=K$. [See Eq. (2.1).] The computation of this gauge invariant quantity from $\psi(r)$ is most easily described in the RW gauge, where $I_{\text {conf }}$ reduces to $H_{2}-K$. From Eq. (2.2) in the RW gauge, and from Eq. (2.18), which is already in the RW gauge, it follows that (for $r \neq r_{p}$ )

$$
K=\frac{6 M^{2}+3 M \lambda r+\lambda(\lambda+1) r^{2}}{r^{2}(\lambda r+3 M)} \psi+\left(1-\frac{2 M}{r}\right) \frac{\partial \psi}{\partial r} .
$$

From this result for $K$, and from Eq. (2.2) in the RW gauge, one finds $H_{2}$ to be (for $r \neq r_{p}$ )

$$
H_{2}=-\frac{9 M^{3}+9 \lambda M^{2} r+3 \lambda^{2} M r^{2}+\lambda^{2}(\lambda+1) r^{3}}{r^{2}(\lambda r+3 M)^{2}} \psi+\frac{3 M^{2}-\lambda M r+\lambda r^{2}}{r(\lambda r+3 M)} \partial_{r} \psi+(r-2 M) \partial_{r}^{2} \psi .
$$

The difference between the expressions in Eqs. (2.26) and (2.25) gives $I_{\text {conf }}$, the gauge invariant measure of the deviation of the 3-geometry from conformal flatness.

\section{Hypersurface data for $\dot{\psi}$}

The integration of the wave equation (2.4), to evolve forward in time from a $t=$ constant hypersurface, requires that we specify both $\psi$ and $\dot{\psi}$ at the hypersurface. In Paper I our initial hypersurface was one of time symmetry, so we had $\dot{\psi}=0$ initially. We now take the particles to be moving on the hypersurface, and for the hypersurface value of the extrinsic curvature we follow the prescription of Bowen and York [14]. In that prescription one chooses a slicing such that the trace $K_{i}^{i}$ of the extrinsic curvature vanishes, and one defines a quantity $\widehat{K}_{i j}$, related to the extrinsic curvature $K_{i j}$ by

$$
K_{i j}=\Phi^{-2} \widehat{K}_{i j}
$$

where $\gamma_{i j}^{\text {flat }}$ is the flat 3 -metric. The curvature measure $\widehat{K}_{i j}$ is considered to be a tensor in the conformally related flat space; its indices are raised and lowered with $\gamma_{i j}^{\text {flat }}$. The momentum constraint turns out to be equivalent to the requirement that $\widehat{\nabla}_{i} \widehat{K}^{i j}$ vanish outside the sources, which we take to be points in the conformally flat 3-geometry. The Bowen-York prescription for initial data is that $\widehat{K}_{i j}$ be "longitudinal," i.e., derivable from a vector $\widehat{W}_{i}$ according to

$$
\widehat{K}_{i j}=\widehat{\nabla}_{i} \widehat{W}_{j}+\widehat{\nabla}_{j} \widehat{W}_{i}-(2 / 3) \gamma_{i j}^{\mathrm{flat}} \widehat{\nabla}_{k} \widehat{W}^{k}
$$


Our choice to describe a moving hole is a solution closely related to those used in most numerical relativity simulations. To describe these solutions we use the geometry of the conformally related flat space pictured in Fig. 11. The symmetry axis is taken to be the $\bar{z}$ coordinate axis and the perturbative particle is located at $\bar{z}=\bar{z}_{p}$. The vectors $\vec{r}$ and $\vec{\rho}$ are the displacements (in the flat space) to a field point, respectively from the coordinate origin and from the location of the moving "particle." The unit vector $\vec{n}$, defined to be $\overrightarrow{\bar{\rho}} /|\overrightarrow{\bar{\rho}}|$ is the direction to the field point from the moving particle. In terms of the notation in this figure, we define two solutions of the momentum constraint $\widehat{\nabla}_{i} \widehat{K}^{i j}=0$. The first, which we shall call the basic solution, $\widehat{K}^{\text {basic }}$, is

$$
\widehat{K}_{i j}^{\mathrm{basic}}=\frac{3}{2 \bar{\rho}^{2}}\left[P_{i} n_{j}+P_{j} n_{i}-\left(\gamma_{i j}^{\mathrm{flat}}-n_{i} n_{j}\right) P^{k} n_{k}\right] .
$$

This is a longitudinal solution that corresponds to the vector

$$
\widehat{W}_{i}^{\text {basic }}=-\frac{1}{4 \bar{\rho}}\left[7 P_{i}+n_{i} P^{k} n_{k}\right] .
$$

The parameter $\vec{P}$ is the momentum of a moving hole in the conformally related flat space. For our particle, $\vec{P}$ is related to the true 3 -momentum (i.e., the spatial part of the 4 -momentum) $\vec{p}$ by $|\vec{P}|=\Phi^{2}|\vec{p}|$. Here $\Phi$ is the conformal factor and, to lowest order, has the form $1+M / 2 \bar{r}$, as in Eq. (2.16). In our head on collision the only nonvanishing component of momentum is the radial component, so we have

$$
P \equiv|\vec{P}|=\Phi^{2} p^{r} / \sqrt{1-2 M / r}
$$

with $p^{r} \equiv m_{0} U^{r}$ the usual contravariant radial component of the 4-momentum in the Schwarzschild background coordinates. This relationship can be verified by using the extrinsic curvature given by Eq. (2.29) in the momentum constraint $R_{t r}=8 \pi T_{t r}$ and taking the right hand side to be the stress energy of a radially moving particle.

The "basic" solution in Eq. (2.29) is the simplest solution of the momentum constraints. It is straightforward, though tedious, to decompose the conformal extrinsic curvature into spherical harmonics. To express the results we adopt the following notation, analogous to that of Regge and Wheeler [f] for the metric perturbations:

$$
\begin{aligned}
\widehat{K}_{\bar{r} \bar{r}} & =\sum_{\ell=1,2, \cdots} \mathcal{A}_{\ell}\left(\bar{r} ; \bar{z}_{p}\right) Y_{\ell 0} \\
\widehat{K}_{\bar{r} \theta} & =\sum_{\ell=1,2, \cdots} \mathcal{B}_{\ell}\left(\bar{r} ; \bar{z}_{p}\right) \partial Y_{\ell 0} / \partial \theta \\
\widehat{K}_{\theta \theta} & =\bar{r}^{2} \sum_{\ell=1,2, \cdots}\left[\mathcal{K}_{\ell}\left(\bar{r} ; \bar{z}_{p}\right) Y_{\ell 0}+\mathcal{G}_{\ell}\left(\bar{r} ; \bar{z}_{p}\right) \partial Y_{\ell 0} / \partial^{2} \theta^{2}\right] \\
\widehat{K}_{\phi \phi} & =\bar{r}^{2} \sin ^{2} \theta \sum_{\ell=1,2, \cdots}\left[\mathcal{K}_{\ell}\left(\bar{r} ; \bar{z}_{p}\right) Y_{\ell 0}+\cot \theta \mathcal{G}_{\ell}\left(\bar{r} ; \bar{z}_{p}\right) \partial Y_{\ell 0} / \partial \theta\right] .
\end{aligned}
$$

Other components vanish due to the azimuthal symmetry.

For the expansion of the basic extrinsic curvature, the explicit expressions for the coefficient functions are

$$
\begin{aligned}
\mathcal{A}_{\ell}^{\text {basic }} & =\sqrt{\frac{4 \pi}{2 \ell+1}}\left(\frac{P}{2 \bar{r}^{2}}\right) q^{l-1}(l+1)(l+2)\left[-\frac{l}{2 l-1}+q^{2} \frac{(l-6)}{2 l+3}\right] \\
\mathcal{B}_{\ell}^{\text {basic }} & =\sqrt{\frac{4 \pi}{2 \ell+1}}\left(\frac{P}{2 \bar{r}}\right) q^{l-1}(l+2)\left[\frac{(l-2)}{2 l-1}-q^{2} \frac{(l-6)}{2 l+3}\right] \\
\mathcal{G}_{\ell}^{\text {basic }} & =\sqrt{\frac{4 \pi}{2 \ell+1}}\left(\frac{P}{2 \bar{r}^{2}}\right) q^{l-1}(l+1)\left[\frac{5 l}{2 l-1}-q^{2} \frac{(l-6)}{2 l+3}\right] \\
\mathcal{K}_{\ell}^{\text {basic }} & =\sqrt{\frac{4 \pi}{2 \ell+1}}\left(\frac{P}{2 \bar{r}^{2}}\right) q^{l-1}\left[-\frac{(l-8)}{2 l-1}+q^{2} \frac{(l-6)}{2 l+3}\right] .
\end{aligned}
$$

Here $q \equiv \bar{z}_{p} / \bar{r}<1$ and for $q>1$, the above expressions are valid if $\ell$ is replaced throughout by $-\ell-1$ everywhere on the righthand side, except in the normalization constant $\sqrt{4 \pi / 2 \ell+1}$.

The parameter $P$ is taken to represent the (perturbative) momentum of the particle. We take our asymptotically flat coordinate system to be one in which there is no net momentum. This means that the large "background" hole 
must have momentum which is in some sense equal in magnitude to the momentum of the particle. If we take the origin of our coordinate system, in the conformally flat space, to be (in some sense) at the center of mass, then the coordinate singularity representing the background hole is at a coordinate distance $\bar{z}_{b h}=-\left(m_{p} / M\right) \bar{z}_{p}$ from the origin. We can get the contribution to the extrinsic curvature from the background hole by reversing the sign of $P$ in the expressions above, and by substituting $\bar{z}_{b h}$ in place of $\bar{z}_{p}$. But $z_{b h}$ is a first order quantity, so $\left(\bar{z}_{b h}\right)^{n}$ terms can be kept only when $n=0$. From Eq. (2.36)-(2.39) we see that this means that only $\ell=1$ terms can be kept. But the $\ell=1$ even parity terms have no physical content, and are not coupled to gravitational radiation. In treating the perturbative extrinsic curvature, therefore, we consider only the contributions from the particle.

To use the above results in calculations, we must relate the extrinsic curvature to the Moncrief wave function $\psi$. It is useful to have at hand the relations between the extrinsic curvature and the metric perturbations. This is found by considering a $t=$ constant slice of the perturbed spacetime. It is straightforward to calculate the extrinsic curvature of this slice in terms of the perturbations of the spacetime metric. In the RW notation (but not the RW gauge), the results are

$$
\begin{aligned}
& \mathcal{A}=\Phi^{-2}\left[(1-2 M / r)^{-1 / 2} H_{1}^{\prime}-\frac{1}{2}(1-2 M / r)^{-3 / 2} \dot{H}_{2}+(1-2 M / r)^{-3 / 2}\left(M / r^{2}\right) H_{1}\right] \\
& \mathcal{B}=\Phi^{-2} \frac{1}{2}(1-2 M / r)^{-1 / 2}\left[H_{1}+h_{0}^{\prime}-\dot{h}_{1}-2 h_{0} / r\right] \\
& \mathcal{K}=\Phi^{-2}\left[r(1-2 M / r)^{1 / 2} H_{1}-\frac{1}{2} r^{2}(1-2 M / r)^{-1 / 2} \dot{K}\right] \\
& \mathcal{G}=\Phi^{-2}(1-2 M / r)^{-1 / 2}\left[h_{0}-\frac{1}{2} r^{2} \dot{G}\right]
\end{aligned}
$$

where prime indicates derivate with respect to $r$, and dot with respect to $t$.

To find the initial value of $\dot{\psi}$, we use the prescription given by Abrahams and Price [15, and we write

$$
\psi=\mathcal{Q}\left\{\delta g_{i j}, \delta g_{i j}^{\prime}\right\}
$$

in which " $\mathcal{Q}$ " represents Moncrief combination [6] of perturbations of the metric $\delta g_{i j}$, and of the $r$ derivative of $\delta g_{i j}$. The initial value of $\dot{\psi}$ is then the initial value of

$$
\dot{\psi}=-2 \mathcal{Q}\left\{(1-2 M / r)^{1 / 2} \delta K_{i j},\left((1-2 M / r)^{1 / 2} \delta K_{i j}\right)^{\prime}\right\}
$$

By comparing Eqs. (2.1) and (2.32)-(2.35) we find that the substitutions needed are:

$$
\begin{aligned}
(1-2 M / r)^{-1} H_{2} & \longrightarrow \Phi^{-2} \mathcal{A}(\partial \bar{r} / \partial r)^{2} \sqrt{1-2 M / r} \\
K & \longrightarrow \Phi^{-2} \mathcal{K}(\bar{r} / r)^{2} \sqrt{1-2 M / r} \\
G & \longrightarrow \Phi^{-2} \mathcal{G}(\bar{r} / r)^{2} \sqrt{1-2 M / r} \\
h_{1} & \longrightarrow \Phi^{-2} \mathcal{B}(\bar{r} / r) \sqrt{1-2 M / r}
\end{aligned}
$$

With these substitutions made in Eq. (2.2) we get a specific expression for $\dot{\psi}$, as a function of $r$, on the hypersurface. An independent derivation of the result starts with the expression

$$
\dot{\psi}(r, t)=\frac{r}{\lambda+1}\left[\dot{K}+\frac{r-2 M}{\lambda r+3 M}\left\{\dot{H}_{2}-r \partial \dot{K} / \partial r\right\}\right]+(r-2 M)\left(r^{2} \partial \dot{G} / \partial r-2 \dot{h}_{1}\right)
$$

where dots indicate $\partial / \partial t$. With Eqs. (2.40)-(2.43). the right hand side of Eq. (2.50) can be reexpressed in terms of $\mathcal{A}, \mathcal{B}, \mathcal{K}, \mathcal{G}$. We have checked that the result is identical to that found from Eqs. (2.45)-(2.49), i.e.

$$
\begin{aligned}
& \dot{\psi}(r, t)=-\frac{2 \bar{r} \sqrt{1-2 M / r}}{(\lambda+1) r^{2}}\left\{\bar{r}^{2} \mathcal{G}+\frac{\sqrt{1-2 M / r}}{(2 \lambda+6 M / r)}\left[-4(\lambda+1)\left(\bar{r}^{2} \mathcal{B}\right)+\frac{M}{r}\left(\frac{1}{\sqrt{1-2 M / r}}+\sqrt{\frac{r}{\bar{r}}}\right)\left(\bar{r}^{2} \mathcal{A}\right)\right.\right. \\
& \left.\left.+\bar{r} \partial_{\bar{r}}\left(\bar{r}^{2} \mathcal{A}\right)\right]\right\}
\end{aligned}
$$

In describing the general choice of $\dot{\psi}$ on a constant $t$ hypersurface, it is useful to review the equivalent question for $\psi$. If we restrict our choice to a conformally flat 3-geometry, then the hamiltonian constraint, and the constraint of 
conformal flatness, reduces the specification of the initial geometry, for each multipole, to a single ordinary differential equation, Eq. (2.18) with $H_{2}=K$. The only choice to be made is the constant, specifying how much of the physically well-behaved homogeneous solution $(2.20)$ is to be added to a particular solution; specifying this constant is equivalent to choosing between, for example, the nonsymmetric (Brill-Lindquist) solution of Eq. (2.12) and the reflection symmetric (Misner) solution of Eq. (2.13). If, on the other hand, we do not restrict ourselves to a conformally flat 3-geometry, we have a functional degree of freedom in the initial geometry. (Equivalently, in the RW gauge we have the freedom to specify $\mathrm{H}_{2}-K$ for each multipole.) Whether or not we restrict to a conformally flat 3-geometry, the Moncrief wavefunction $\psi$, defined in Eq. (2.2), is fixed once the 3 -geometry is specified. Furthermore, $\psi$, is totally gauge invariant.

For $\dot{\psi}$ the situation is closely parallel. Analogous to the condition on 3-geometry, that it be conformally flat, we now have the special condition that the extrinsic curvature be purely "longitudinal," i.e., derivable from a potential according to Eqs. (2.27) and (2.28). For our axisymmetric infall, the mathematical problem of finding the $\widehat{W}_{i}$ potential, for each multipole, is the problem of finding the two components $\widehat{W}_{\bar{r}}$ and $\widehat{W}_{\theta}$. These can be expanded in multipoles and written as:

$$
\widehat{W}_{\bar{r}}=\sum_{\ell} A_{\ell}(\bar{r}) Y_{\ell 0} \quad \widehat{W}_{\theta}=\sum_{\ell} B_{\ell}(\bar{r}) \partial Y_{\ell 0} / \partial \theta
$$

The nontrivial momentum constraints, equivalent to the $t \theta$ and $t r$ components of the einstein equations, then constitute two second order differential equations for $A_{\ell}(\bar{r})$ and $B_{\ell}(\bar{r})$. A particular solution to the momentum constraints is, of course, given by the fundamental solution in Eq. (2.29). This solution corresponds to the $\widehat{W}^{i}$ multipoles

$$
\begin{gathered}
A_{\ell}^{\text {basic }}(\bar{r})=\sqrt{\frac{4 \pi}{2 \ell+1}} \frac{P}{4 \bar{r}}\left[\frac{\ell(\ell+7)}{2 \ell-1} q^{\ell-1}+\frac{(\ell+1)(6-\ell)}{2 \ell+3} q^{\ell+1}\right] \\
B_{\ell}^{\text {basic }}(\bar{r})=\sqrt{\frac{4 \pi}{2 \ell+1}} \frac{P}{4}\left[\frac{(8-\ell)}{2 \ell-1} q^{\ell-1}+\frac{(\ell-6)}{2 \ell+3} q^{\ell+1}\right] .
\end{gathered}
$$

The above expressions are valid only for $q \equiv \bar{z}_{p} / \bar{r} \leq 1$. For $q>1$ the replacement $\ell \rightarrow-\ell-1$ must be made inside the square brackets.

One finds that there are only two solutions well behaved at infinity, and that all solutions are well behaved at the horizon. There are then two well-behaved homogeneous solutions of the momentum constraints that can be added to the particular solution of Eqs. (2.53) and (2.54).

Homogeneous longitudinal solution to the momentum constraints can be found directly from the differential equations. Alternatively one can construct those from known solutions. One such solution is an "image solution," the basic solution given in Eq. (2.30), but with $\bar{z}_{p}$ replaced by the location $\bar{z}_{\text {image }}<M / 2$, so that the position of the $\bar{z}=\bar{z}_{\text {image }}$ singularity is inside the horizon of the background hole. (If the choice $z_{\text {image }}=(M / 2) / \bar{z}_{p}$ is made, then the singularity is the "image" of the particle under inversion through the spherical surface at $\bar{r}=M / 2$.) The image solution is, then, the following simple modification of Eq. (2.53) and (2.54):

$$
\begin{gathered}
A_{\ell}^{\text {image }}(\bar{r})=\sqrt{\frac{4 \pi}{2 \ell+1}} \frac{P}{4 \bar{r}}\left[\frac{\ell(\ell+7)}{2 \ell-1} q_{\text {image }}^{\ell-1}+\frac{(\ell+1)(6-\ell)}{2 \ell+3} q_{\text {image }}^{\ell+1}\right] \\
B_{\ell}^{\text {image }}(\bar{r})=\sqrt{\frac{4 \pi}{2 \ell+1}} \frac{P}{4}\left[\frac{(8-\ell)}{2 \ell-1} q_{\text {image }}^{\ell-1}+\frac{(\ell-6)}{2 \ell+3} q_{\text {image }}^{\ell+1}\right]
\end{gathered}
$$

Here $q_{\text {image }} \equiv \bar{z}_{\text {image }} / \bar{r}$ and is always less than unity for $\bar{r}$ outside the horizon (i.e., $\bar{r}>M / 2$ ). Similarly, we can find the multipole decomposition of the corresponding extrinsic curvature, simply by replacing $q$ by $q_{\text {image }}$ in Eqs. (2.36) $-(2.39)$.

A second longitudinal solution can be constructed from the " $\alpha$ " solution given by Bowen and York 14.16] and elsewhere:

$$
\widehat{K}_{i j}^{\alpha}=\frac{3}{2 \bar{\rho}^{4}}\left[P_{i} n_{j}+P_{j} n_{i}+\left(\gamma_{i j}^{\text {flat }}-5 n_{i} n_{j}\right) P^{k} n_{k}\right]
$$

The equivalent $\widehat{W}_{i}$ vector is 


$$
\widehat{W}_{i}^{\alpha}=-\frac{1}{4 \bar{\rho}^{3}}\left[P_{i}-3 n_{i} P^{k} n_{k}\right] .
$$

This solution has been of interest in connection with inversion symmetry, but we use it here with no prejudice about symmetry of the solution under inversion. To make the solution of Eqs. (2.57) and (2.58) a homogeneous solution we need only place the $\bar{\rho}=0$ singularity inside the horizon, say on the $\bar{z}$ axis at $\bar{z}_{\alpha}<M / 2$.

The multipole decomposition of the $\alpha$ solution, in the notation of Eqs. (2.32) - (2.35) is

$$
\begin{aligned}
\mathcal{A}_{\ell}^{\alpha} & =\sqrt{\frac{4 \pi}{2 \ell+1}} \frac{P}{2 \bar{r}^{4}} \ell(\ell+1)(\ell+2) q_{\alpha}^{\ell-1} \\
\mathcal{B}_{\ell}^{\alpha} & =-\sqrt{\frac{4 \pi}{2 \ell+1}} \frac{P}{2 \bar{r}^{3}} \ell(\ell+2) q_{\alpha}^{\ell-1} \\
\mathcal{G}_{\ell}^{\alpha} & =\sqrt{\frac{4 \pi}{2 \ell+1}} \frac{P}{2 \bar{r}^{4}} \ell q_{\alpha}^{\ell-1} \\
\mathcal{K}_{\ell}^{\alpha} & =-\sqrt{\frac{4 \pi}{2 \ell+1}} \frac{P}{2 \bar{r}^{4}} \ell(\ell+1) q_{\alpha}^{\ell-1},
\end{aligned}
$$

where $q_{\alpha} \equiv \bar{z}_{\alpha} / \bar{r}$ and is always less than unity for $\bar{r}$ outside the horizon. For the $\alpha$ solution, the multipole decomposition of the $\widehat{W}_{i}$ vector, in the notation of Eq. (2.52), is

$$
\begin{gathered}
A_{\ell}^{\alpha}(\bar{r})=-\sqrt{\frac{4 \pi}{2 \ell+1}} \frac{P}{4 \bar{r}^{3}} \ell(\ell+1) q_{\alpha}^{\ell-1} \\
B_{\ell}^{\alpha}(\bar{r})=\sqrt{\frac{4 \pi}{2 \ell+1}} \frac{P a}{4 \bar{r}^{2}} \ell q_{\alpha}^{\ell-1} .
\end{gathered}
$$

The general longitudinal solution for the extrinsic curvature, for each multipole, is a superposition of three contributions: (i) the particular solution given by Eqs. (2.36)-(2.39), (ii) the homogeneous "image" solution corresponding to Eqs. (2.55), (2.56), for some $\ell$-dependent choice of $\bar{z}_{\text {image }}$, multiplied by an arbitrary, $\ell$-dependent constant, and (iii) the homogeneous " $\alpha$ " solution in Eqs. (2.57) - (2.64), for some $\ell$-dependent choice of $\bar{z}_{\alpha}$, multiplied by an arbitrary, $\ell$-dependent constant. One is led to ask what the "proper" choice is for the general longitudinal solution. A somewhat broader question is whether the initial data should be chosen to be longitudinal.

In the initial data solutions generated for use in numerical relativity, the choice has often been made to take the solution to be inversion symmetric [17]. (That solution turns out not, in fact, to be longitudinal.) The criterion used in numerical relativity has been definiteness and the simple-to-implement inner boundary condition provided by inversion symmetry. Here our criterion shall be quite different: we study what choice of hypersurface data is the best representation of the solution that evolves from an earlier astrophysical configuration. More specifically, we can start a particle falling from some large radius $r_{0}$, and numerically evolve $\psi$ to a hypersurface at some later time. We can then study the agreement between that evolved data and the data given by some prescription for assigning hypersurface data to represent the falling particle.

It should be understood that in making this comparison (i.e., evolved data vs. prescribed data) there are no issues of "choice" involving the slicing of spacetime. The Moncrief wave variable $\psi$ is invariant with respect to perturbative changes in slicing (as well as shifts and diffeomorphism), so that our comparison of evolved $\psi$ and prescribed $\psi$ involves no gauge related ambiguities.

\section{Choice of hypersurface data}

In our discussion above of initial data we found the following: (i) If we restrict ourselves to conformally flat data, we must choose a single constant, for each multipole, to specify our solution for $\psi$; if we do not restrict to conformally flat data there is an unconstrained function of $r$ for each initial multipole of $\psi$. (ii) If we restrict our choice to longitudinal initial extrinsic curvature, then we must specify two constants for each multipole, in order to fix the initial $\dot{\psi}$; if we do not restrict to initial longitudinal extrinsic curvature, then there is an unconstrained function of $r$ for each multipole of $\dot{\psi}$ (in our case of axially symmetric collision).

Our goal below will be to see whether there is a simple physical choice that can be made for the prescribed hypersurface data that agrees well with the data evolved from $t=0$. We shall try to reach that goal by restricting 
our prescription to conformally-flat, longitudinal (hereafter CFL) data. The question we shall be asking, therefore, is whether there exists a choice of constants specifying the CFL solution for the particle on a hypersurface so that it agrees well with an evolved solution. Such a choice of constants could be determined by looking for some kind of "best fit" of the CFL solution to the evolved solution, but of much greater interest is a physically-based prescription for the choice of constants which can be used as a best guess of appropriate initial data when an evolved solution is not available.

The rule we shall use for the specification of the CFL $\psi$ is based on a simple physical consideration. We fix the constant in the CFL solution for $\psi$ by demanding that the value of $\psi$ at the horizon be "frozen," i.e., fixed at the value it has on the initial hypersurface $t=0$. This is in accord with the idea of dynamics freezing at the horizon, but it is better to view it as a consequence of the wave equation Eq. (2.4), and its horizon limit at $r^{*} \rightarrow-\infty$. The initial data at $t=0$ is a smooth function of $\bar{r}$ near the horizon, and hence $\psi$ becomes constant as $r^{*} \rightarrow-\infty$. But the potential decreases as $e^{r^{*} / 2 M}$ and can be ignored near the horizon, so $\psi=$ constant is a static solution of the wave equation. The value of $\psi$ at the horizon will therefore be unchanging in time. We shall see, in the numerical results presented below, that the evolved data demonstrate this freezing.

It is straightforward to use this condition of freezing at the horizon to fix the constant multiplier of the homogeneous solution in Eq. (2.20), and the consequence is interesting. If we start with a BL solution (i.e., the solution in Eq. (2.19) with the particle position at $\bar{z}_{p}=\bar{r}_{0}$ ) then the "horizon frozen" solution, on a later hypersurface, turns out to be a superposition of the following simple solutions: (i) the BL solution corresponding to the the particle at its correct position $\bar{z}_{p}$ at time $t$, (ii) a solution for a particle inside the horizon located at $\bar{z}_{\text {image }}=(\mathrm{m} / 2)^{2} / \bar{z}_{p}$, so that when added to the BL solution the total value of $\psi$ vanishes at the horizon, and (iii) a solution corresponding to a particle at the image location of the original position $\bar{z}=(M / 2)^{2} / \bar{r}_{0}$. The magnitude of the mass parameter for this last image gives a $\psi$ at the horizon equal to the frozen value.

The way in which we fix the constants for $\dot{\psi}$ is a more complicated, less elegant, implementation of the same basic physical idea of looking at the nature of solutions of Eq. (2.4), near the horizons. At $t=0$ our initial data is taken to be stationary, and of the form

$$
\psi=a+b \bar{r}+c \bar{r}^{2}+\cdots
$$

Since $\bar{r}$ is related to $r^{*}$ by

$$
\bar{r}=M / 2+M e^{-1 / 2} e^{r^{*} / 4 M}+M e^{-1} e^{r^{*} / 4 M}+\mathcal{O}\left(e^{3 r^{*} / 4 M}\right),
$$

it follows that our initial solution has the horizon limit

$$
\psi=\tilde{a}+\tilde{b} e^{r * / 4 M}+\tilde{c} e^{r * / 2 M}, \quad \dot{\psi}=0 .
$$

In the horizon limit, the potential $V_{\ell}$, in Eq. (2.4), takes the form

$$
V_{\ell}=e^{r^{*} / 2 M} V_{o}+\mathcal{O}\left(e^{r^{*} / M}\right), \quad V_{o}=\frac{4 \lambda^{2}+4 \lambda+3}{(2 \lambda+3) 4 M^{2} e}
$$

where $V_{o}$ is a constant. In the horizon limit we are, then, solving the problem

$$
-\frac{\partial^{2} \psi}{\partial t^{2}}+\frac{\partial^{2} \psi}{\partial r *^{2}}-e^{r^{*} / 2 M} V_{o} \psi=0
$$

with the initial data given by Eq. (2.67). It is simple to show that the solution to this problem is

$$
\psi=\tilde{a}+\tilde{b} \cosh (t / 4 M) e^{r^{*} / 4 M}+\left[\left(\tilde{c}-V_{o}(2 M)^{2} \tilde{a}\right) \cosh (t / 2 M)+V_{o}(2 M)^{2} \tilde{a}\right] e^{r^{*} / 2 M} .
$$

We can now take the time derivative of this near-horizon solution, evaluate it on the hypersurface at time $t$, and find

$$
\left.\dot{\psi}\right|_{t}=(\tilde{b} / 4 M) \sinh (t / 4 M) e^{r^{*} / 4 M}+\left(\tilde{c}-V_{o}(2 M)^{2} \tilde{a}\right) \sinh (t / 2 M) e^{r^{*} / 2 M} .
$$

This result can be compared with the Taylor expansion around $\bar{r}=M / 2$ of the "basic," "image," and " $\alpha$ " longitudinal solutions, and the two constants fixing the solution thereby determined.

From Eq. (2.70) it can be immediately seen that $\tilde{a}$ does not depend on time. This is equivalent to the condition of "freezing" on the horizon. The amplitude $\Gamma$ by which we have to multiply the homogeneous solution (2.20) is

$$
\Gamma_{\text {homog }}\left(\bar{z}_{p}\right)=\frac{m_{2}\left(r_{0}\right)}{m_{2}\left(z_{p}\right)}\left(\frac{\bar{z}_{p}}{\bar{r}_{0}}\right)^{l+1}\left(1+\Gamma_{\text {homog }}\left(r_{0}\right)\right)-1
$$


where $\Gamma_{\text {homog }}\left(r_{0}\right)$ represents the arbitrariness in choosing the data on the initial hypersurface $t=0$. For instance, $\Gamma_{\text {homog }}\left(r_{0}\right)=0,1,-1$ represents respectively BL, Misner, and antisymmetric data.

The amplitude of the "image" term in the extrinsic curvature is the following

$$
\begin{aligned}
\Gamma_{\text {image }}= & \Delta^{-1}\left\{\frac{4 q_{3}^{l+2} q_{4}^{l-1}}{M^{2}}\left[\frac{6 l(4 l+1)}{2 l+3}\right]\right. \\
& +\frac{16 m_{p}\left(r_{0}\right)(2 l+1) q_{4}^{2 l}}{M^{2} P}\left[4 l\left(1-\Gamma_{\text {homog }}\left(r_{0}\right)\right) \sin (t / 4 M)\right. \\
& \left.\left.+\frac{\left(1+\Gamma_{\text {homog }}\left(r_{0}\right)\right)\left(5-l-3 l^{2}-4 l^{3}-2 l^{4}\right)}{(l+1)\left(l^{2}+l+1\right)} \sin (t / 2 M)\right]\right\},
\end{aligned}
$$

where

$$
\Delta=\frac{4 q_{3}^{l-1} q_{4}^{l-1}}{M^{2}}\left(\frac{2 l}{2 l+3}\right)\left[-(5 l+1)(2 l+3)+(l-6)(2 l+1) q_{3}^{2}\right],
$$

$\bar{z}_{\text {image }}=(M / 2)^{2} / \bar{z}_{p}, q_{3}=M /\left(2 \bar{z}_{p}\right)$, and $q_{4}=M /\left(2 \bar{r}_{0}\right)$.

Finally, the amplitude of the $\alpha$ term in the extrinsic curvature (see Eq. (2.57)) is

$$
\begin{aligned}
\Gamma_{\alpha}= & \Delta^{-1}\left\{2 q_{3}^{2 l-1}\left[\frac{-(l+7)(5 l+1)}{2 l-1}+\frac{(2 l+1)\left(26 l^{2}+26 l-27\right)}{(2 l-1)(2 l+3)} q_{3}^{2}-\frac{(l-6)(5 l+4)}{2 l+3} q_{3}^{4}\right]\right. \\
& +\frac{16 m_{2}\left(r_{0}\right)\left(1-\Gamma_{h o m o g}\left(r_{0}\right)\right)(2 l+1) q_{3}^{l-1} q_{4}^{l+1}}{P}\left[-\frac{5(l-2)}{2 l-1}+\frac{(l-6)}{2 l+3} q_{3}^{2}\right] \sin (t / 4 M) \\
& \left.+\frac{4 m_{2}\left(r_{0}\right)\left(1+\Gamma_{\text {homog }}\left(r_{0}\right)\right) q_{3}^{l-1} q_{4}^{l+1}}{l(l+1)\left(l^{2}+l+1\right) P}\left[-\frac{10 l^{2}+9 l+8}{2 l-1}+\frac{(l-6)(2 l+1)}{2 l+3} q_{3}^{2}\right] \sin (t / 2 M)\right\},
\end{aligned}
$$

where $\bar{z}_{\alpha}=(M / 2)^{2} / \bar{r}_{0}$.

Note that this choice of the amplitudes we assign to the homogeneous solutions fixes all three constants in the general solution, but this is not the only way we can fix them. We could have chosen, for instance, matching the behavior of the evolved $\psi$ for large $r$. This, however, leads to a less successful approximation.

\section{RESULTS}

\section{A. Numerical method}

In this section we describe the algorithm used to integrate the wave equation (2.4) numerically. While the left hand side of the equation is straightforward to integrate, the source given by Eq. (2.8) contains terms with a Dirac's delta and its derivative. Since we have not found in the literature a discussion of the numerical treatment of such sources, we shall describe the method in some detail.

We have found it convenient to use a numerical scheme with step sizes $\Delta t=\frac{1}{2} \Delta r^{*} \equiv h$, and with a staggered grid. As Fig. 2 shows, this method connects points along lines of constant "retarded time" $u \equiv t-r^{*}$ and "advanced time" $v \equiv t+r^{*}$. On this grid we have implemented a finite difference algorithm for evolving $\psi$ with errors of order $h^{2}$; that is, at a given value of $t$ and $r *$ a measure of the solution error varies as $h^{2}$. This method has proven to be easy to implement and quite accurate.

To derive our difference scheme we start by integrating Eq. (2.4) over the cell of our numerical grid shown in Fig. 2. We use the notation

$$
\iint d A=\iint_{\text {cell }} d t d r^{*}=\int_{u-h}^{u+h} d u \int_{v-h}^{v+h} d v
$$

Applied to the derivative terms in Eq. (2.4) this gives:

$$
\begin{aligned}
& \iint d A\left\{-\partial_{t} \partial_{t} \psi+\partial_{r}^{*} \partial_{r}^{*} \psi\right\}=\iint d A\left\{-4 \partial_{u} \partial_{v} \psi\right\}= \\
& -4\left[\psi\left(t+h, r^{*}\right)+\psi\left(t-h, r^{*}\right)-\psi\left(t, r^{*}+h\right)-\psi\left(t, r^{*}-h\right)\right] .
\end{aligned}
$$


Note that this result is exact; it contains no truncation errors.

We next consider the integration of the potential term over the cell. If the cell is one with no source term contribution, then we can use

$$
\begin{aligned}
& \iint d A\{-V \psi\}=-h^{2}\left[V\left(r^{*}\right) \psi\left(t+h, r^{*}\right)+V\left(r^{*}\right) \psi\left(t-h, r^{*}\right)+\right. \\
& \left.V\left(r^{*}+h\right) \psi\left(t, r^{*}+h\right)+V\left(r^{*}-h\right) \psi\left(t, r^{*}-h\right)\right]+\mathcal{O}\left(h^{4}\right) .
\end{aligned}
$$

The $h^{4}$ order error in a generic cell is equivalent to an overall $\mathcal{O}\left(h^{2}\right)$ error in $\psi$.

The result in Eq. (3.3) assumes that $\psi$ is smooth in the grid cell. It cannot be applied to those cells through which the particle worldline passes, since $\psi$ is discontinuous across the worldline. For such cells we first obtain the coordinates $\left(r_{1}^{*}, t_{1}\right)$ of the point where the particle enters the cell and $\left(r_{2}^{*}, t_{2}\right)$ where the particle leaves it (see Fig. 2). Next, we divide the total area of the cell, $\left(4 h^{2}\right)$, into four subareas defined as follows: $A_{2}$ is the part of the area of the rhomb below $t=t_{1}, A_{3}$ is the part of the area of the rhomb over $t=t_{2}, A_{1}$ is the remaining area to the left of the particle's trajectory, and $A_{4}$ is the remaining area to the right.

The integral of the $V \psi$ term over the area of the cell is approximated by the sum of this function evaluated on the corners of the cell multiplied by the corresponding subarea $A_{i}$. This gives us

$$
\iint d A\{-V \psi\}=-V(r *)\left[\psi\left(t+h, r^{*}\right) A_{3}+\psi\left(t, r^{*}+h\right) A_{4}+\psi\left(t, r^{*}-h\right) A_{1}+\psi\left(t-h, r^{*}\right) A_{2}\right] .
$$

The truncation error in each such cell is of order $\left(h^{3}\right)$, just enough to have quadratic convergence, since only one cell with the particle has to be evaluated per time step.

For cells through which the worldline passes, the integral of the source term in Eq. (2.8) must be evaluated. As a convenience in discussing the numerical approximation of this term Eq. (2.8), we introduce the notation

$$
\mathcal{S}=G(t, r) \delta\left[r-r_{p}(t)\right]+F(t, r) \delta^{\prime}\left[r-r_{p}(t)\right]
$$

The integration over the cell, when done with due regard to the boundary terms generated by the $\delta^{\prime}\left[r-r_{p}(t)\right]$, gives

$$
\begin{aligned}
\iint \mathcal{S} d A= & 2 \int_{t_{1}}^{t_{2}} d t\left[\frac{G\left(t, r_{p}(t)\right)}{1-2 M / r_{p}(t)}-\left.\partial_{r}\left(\frac{F(t, r)}{1-2 M / r}\right)\right|_{r=r_{p}(t)}\right] \\
& \pm 2 \frac{F\left(t_{1}, r_{p}\left(t_{1}\right)\right)}{\left(1-2 M / r_{p}\left(t_{1}\right)\right)^{2}}\left(1 \mp \dot{r}_{p}^{*}\left(t_{1}\right)\right)^{-1} \\
& \pm 2 \frac{F\left(t_{2}, r_{p}\left(t_{2}\right)\right)}{\left(1-2 M / r_{p}\left(t_{2}\right)\right)^{2}}\left(1 \pm \dot{r}_{p}^{*}\left(t_{2}\right)\right)^{-1}
\end{aligned}
$$

The $\int d t$ integral in the first term can be performed to any precision since $F$ and $G$ are known functions. For our goal of quadratic convergence, a trapezoidal approximation for the integration is adequate. In the second term the upper (lower) sign is for particles entering the cell from the right (left), or equivalently for $r_{1}^{*}>r^{*}\left(r_{1}^{*}<r^{*}\right)$. In the same way, in the third term the upper (lower) sign is for particles leaving the cell to the right (left), or equivalently $r_{2}^{*}>r^{*}\left(r_{2}^{*}<r^{*}\right)$.

When the form of $F$ and $G$, given in Eq. (2.8), are used in Eq. (3.6) the result is

$$
\begin{aligned}
& \frac{F\left(t, r_{p}(t)\right)}{\left(1-2 M / r_{p}(t)\right)^{2}}=\frac{\kappa \epsilon_{0}^{-1}\left(r_{p}-2 M\right)}{(\lambda+1)\left(\lambda r_{p}+3 M\right)} ; \epsilon_{0} \equiv \sqrt{1-2 M / r_{0}} \\
& \frac{G\left(t, r_{p}(t)\right)}{1-2 M / r_{p}(t)}-\left.\partial_{r}\left(\frac{F(t, r)}{1-2 M / r}\right)\right|_{r=r_{p}(t)}=\frac{\kappa \epsilon_{0}^{-1}\left(1-2 M / r_{p}\right)}{(\lambda+1) r_{p}\left(\lambda r_{p}+3 M\right)^{2}}\left[3 M^{2}-4 \lambda M r_{p}-\lambda(\lambda+1) r_{p}^{2}-6 M\left(1-\epsilon_{0}^{2}\right) r_{p}\right] \\
& \dot{r}_{p}^{*}(t)=-\epsilon_{0}^{-1} \sqrt{2 M / r_{p}-2 M / r_{0}}
\end{aligned}
$$

Our numerical scheme, for cells through which the particle worldline does not pass, is to solve for $\psi\left(t, r^{*}\right)$, using Eq. (3.2) and Eq. (3.3) in the integral of Eq. (2.4). For cells containing the worldline, Eq. (3.2), Eq. (3.4), Eq. (3.6) and Eq. (3.7) are used. In summary, the evolution algorithm we use is

$$
\psi\left(t+h, r^{*}\right)=-\psi\left(t-h, r^{*}\right)+\left[\psi\left(t, r^{*}+h\right)+\psi\left(t, r^{*}-h\right)\right]\left[1-\frac{h^{2}}{2} V\left(r^{*}\right)\right]
$$


for cells not crossed by the particle, and

$$
\begin{aligned}
\psi\left(t+h, r^{*}\right)= & -\psi\left(t-h, r^{*}\right)\left[1+\frac{V\left(r^{*}\right)}{4}\left(A_{2}-A_{3}\right)\right]+\psi\left(t, r^{*}+h\right)\left[1-\frac{V\left(r^{*}\right)}{4}\left(A_{4}+A_{3}\right)\right] \\
& +\psi\left(t, r^{*}-h\right)\left[1-\frac{V\left(r^{*}\right)}{4}\left(A_{1}+A_{3}\right)\right]-\frac{1}{4} \iint \mathcal{S} d A,
\end{aligned}
$$

for the cells that the particle does cross.

The above equations cannot, however, be used to initiate the evolution off the first hypersurface. If $t_{0}$ denotes the time at which we have the initial data, we lack the values $\psi\left(t_{0}-h\right)$, necessary to apply the evolution algorithm. We can, however, use a Taylor expansion to write

$$
\psi\left(t_{0}-h, r^{*}\right)=\psi\left(t_{0}+h, r^{*}\right)-2 h \partial_{t} \psi\left(t_{0}, r^{*}\right)+\mathcal{O}\left(h^{3}\right) .
$$

The right hand side can be used in place of $\psi\left(t_{0}-h, r^{*}\right)$ in the application of the algorithm to evolve off the first hypersurface. It is important to note that this substitution is valid only if $\psi\left(t, r^{*}\right)$ is not singular between $t=t_{0}-h$ and $t=t_{0}-h$. This requires that the particle worldline not cross the vertical line at $r^{*}$ between $t=t_{0}-h$ and $t=t_{0}+h$. In setting up the computational grid, we have been careful always to avoid such a crossing.

The numerical method used here, evolving initial data for a partial differential equation on a staggered grid, has little in common with the transform method used in Paper I, in which we studied only momentarily stationary initial conditions. A comparison of the two methods (in the case of momentarily stationary initial data) provided a powerful check of both methods as well as insights into the relative efficiency and accuracy of the methods. The agreement of the two approaches turned out to be excellent. For the goal of producing an evolved waveform, the numerical evolution method was found to be faster to a transform method by orders of magnitude, and to give more accurate results.

\section{B. Numerical results}

In this section we present computed results for the infall of a particle, starting from rest at initial coordinate position $r_{0}$. The particle trajectory is a radial geodesic as described by Eq. (2.7). By " $t=0$," we shall always mean the hypersurface at which the particle was at rest. We shall consider two values of $r_{0} / 2 M$ : both 15 and 1.5 . The former is a starting point where the influence of the background hole is reasonably weak. (The gravitational redshift, for example, is around only $3 \%$.) This represents, then, an astrophysical starting point for which a Newtonian description would be a good approximation. The second choice, $r_{0} / 2 M=1.5$ is not a reasonable astrophysical starting point, but complements the first choice of $r_{0}$, magnifies certain effects, and is useful for exploration and illustration.

We will be interested in Cauchy data, both $\psi$ and $\dot{\psi}$, on subsequent $t=$ constant hypersurfaces. (We repeat here an important feature of the formalism: the gauge invariant information $\psi$ and $\dot{\psi}$ is unaffected by a change in slicing. When we say, therefore, a $t=$ constant hypersurface, we mean only $t=$ constant to zero order in the particle mass.) There are several ways in which we can specify which later slice we are considering. (i) We could specify the Schwarzschild coordinate time $t$ for this hypersurface. (ii) We could specify the location of the infalling particle corresponding to time $t$; for this purpose we use the notation $r_{p}$ (or $r_{p}^{*}$, the $r^{*}$ equivalent). Note that $r_{p}$ is related to the position parameter $\bar{z}_{p}$ by $r_{p}=\bar{z}_{p}\left(1+M / 2 \bar{z}_{p}\right)^{2}$. (iii) We could specify the parameter $P$ describing the particle's momentum at time $t$. (This is the $P$ parameter that enters into the Bowen-York description of extrinsic curvature; see the discussion in Sec. II relating this parameter to the particle's 4-momentum components. A picture of a sliced Schwarzschild spacetime, illustrating the particle trajectory and the labeling of the spacetime, is shown in Fig. 3 .

In our results the crucial concept is the difference between "evolved" data and "prescribed" data. By "evolved" values of $\psi$ and $\dot{\psi}$ we shall mean the values that are found on some hypersurface after numerical evolution forward in time from the $t=0$ original hypersurface. By "prescribed data" we shall mean data that is chosen according to one of the prescriptions of Sec. II. Prescribed data, for our purposes here, is always chosen from the set of possibilities that we describe as conformally flat, longitudinal. This means that the 3-geometry is conformally flat, and the extrinsic curvature is "longitudinal," in the sense defined by York [18]. For $\psi$, specific choices that we have described in Sec. II include: (i) BL data, the simplest choice for $\psi$, (ii) Misner data, data that is symmetric with respect to reflection under $\bar{r} \rightarrow(M / 2)^{2} / \bar{r}$ in the conformally related flat space, (iii) "frozen" data, the conformally flat solution of the hamiltonian constraint that has the value of $\psi$ at the horizon fixed to be the horizon value on the original $(t=0)$ hypersurface. Prescribed choices for $\dot{\psi}$ include (i) the basic Bowen-York [14] solution, the simplest longitudinal solution, and (ii) "horizon matched" data, the longitudinal solution with the form of $\dot{\psi}$ (more specifically, the first and second derivatives with respect to $r^{*}$ ) matched to the evolved solution at the horizon limit. 
We first present comparisons of radiated energy. In Fig. 4 4 we show the quadrupole energy $E_{2}$ radiated during the infall of the particle from $r_{0}=1.5(2 M)$. On the original $t=0$ hypersurface, the form of $\psi$ is taken to be the Misner solution. (Since the particle is momentarily stationary at $t=0$, we have $\dot{\psi}=0$ on this hypersurface, of course.) The plot shows the energy radiated from the particle for times after the particle is at a location $r^{*}=r_{p}^{*}$. These energies are computed from the solution that evolves from the data on the constant time hypersurface labeled $r_{p}^{*}$. The "true" data are the data that evolved from the $t=0$ original hypersurface on which the particle was momentarily stationary. The energy computed in this way is the "true" total quadrupole energy emitted during infall from $15(2 M)$, and has the value $8.1 \times 10^{-3} m_{0}^{2} /(2 M)$.

The plot shows the energy generated when the true (i.e., evolved) $\psi$ and $\dot{\psi}$, on a hypersurface, are replaced with prescribed data appropriate on that hypersurface for the position and momentum of the geodesically falling particle. To find the points for the dotted curve, for example, on each hypersurface, position and momentum of the infalling particle were calculated and used to generate Misner data for $\psi$, and Bowen-York data for $\dot{\psi}$, on that hypersurface. These prescribed data were then numerically evolved forward in time, and the resulting radiation to a distant observer was computed. This radiated energy is presented as a function of the hypersurface label $r_{p}^{*}$. Similarly, the solid curve shows the result of replacing the true hypersurface data with frozen data for $\psi$ and horizon-matched data for $\dot{\psi}$. The energies of the dashed curve are the result of replacing the $\dot{\psi}$ data with horizon-matched data, and retaining the true $\psi$ data.

These results show that the "frozen-matched" prescription are in slightly better agreement with the correct radiated energy than are the other choices. The choice of frozen-matched conditions is also more justifiable, since it is based on a physical consideration. Throughout the remainder of the paper, we shall consider the frozen-matched choice as the best in the set of conformally flat, longitudinal possibilities.

In Fig. 4 , what is more important than the comparison of prescriptions for hypersurface data is the fact that none of them is very good. Figure 5 gives some insight into the details of the failure. Here, for infall from $r_{0}=1.5(2 M)$, the data for $\psi$ on each hypersurface, are replaced by frozen data; the true data for $\dot{\psi}$ (i.e., the data evolved from $t=0)$ are retained. It is important and interesting that the replacement of the $\psi$ data introduces a much smaller error than the replacement of $\dot{\psi}$. This is a similar conclusion that one finds in the results of Baker et al. [19] in a rather different context.

The previous energy results, of course, are for infall from an astrophysically unreasonable initial radius. In Fig. 6, energy results are shown for infall from $r_{0}=15(2 M)$, in which case the true total quadrupole radiated is $1.64 \times$ $10^{-2} m_{0}^{2} /(2 M)$. Results are shown for three choices of prescribed hypersurface data. Again we see that the agreement with the true energy is reasonably good if the true (i.e., evolved) data for $\dot{\psi}$ is retained. For the other cases the agreement is good only if the data is replaced on a hypersurface well before the particle reaches the peak of the potential $V_{\ell}$ at around $1.55(2 M)$.

We ask next what the appearance of hypersurface data is, and how the true and prescribed forms differ. In Fig. 0 we show the form of the true quadrupole $\psi$ generated by a particle falling from rest at $r_{0}=15(2 M)$, with Misner data initially specified. The figure shows the manner in which $\psi\left(r^{*}\right)$ evolves from its initial Misner form at $t=0$. As the particle moves inward, the simple prescribed form of a single peak evolves into more complex shapes. Figure 8 shows that on late hypersurfaces, after the particle, at $t \approx 100, r_{p}^{*} \approx 0$ has passed through the region in which the Zerilli potential is strong, $\psi$ clearly contains the shape of in- and outgoing waves with the profile of quasinormal ringing.

Figures 9 - 11 compare the true $\psi$ on a hypersurface with prescribed horizon frozen data on the same hypersurface. It should be noted that the magnitude of the discontinuity in the $r$ derivative of $\psi$ is related, through the hamiltonian constraint, to the mass of the particle. It must, therefore, be the same for the pairs of curves in these figures, and for all forms of $\psi$ satisfying the hamiltonian constraint. Results are shown for several different hypersurfaces, and the meaning is clear. The prescribed $\psi$ never contains the complexity of shape that indicates the presence of radiation.

In Fig. 12, $\psi$ is shown for Misner prescribed data on a sequence of hypersurfaces, this time for infall from $r_{0}=$ 1.5 $(2 M)$. In Fig. 13 the frozen prescribed $\psi$ solution is shown on the same hypersurfaces. The comparison of the two figures shows the difference induced by the freezing of the value of $\psi$ at the horizon.

The differences in the evolved and prescribed forms of $\psi$ suggest that at late times the form of $\psi$ is not conformally flat initial data. This is explicitly demonstrated and quantified in Figs. 14 and 15, in which the gauge invariant index of conformality, developed in Sec. II.B is plotted. The traveling bumps, with the appearance of radiation, confirm that the wave content of the true initial data is associated with its failure to be conformally flat.

We next look at results for $\dot{\psi}$. Figure 16, 17, 18 compare the true (i.e., evolved) form of the quadrupolar $\dot{\psi}$ with the horizon matched prescription, for infall from rest (and Misner data) at $t=0, r_{0} / 2 M=15$. As would be expected, the difference between the evolved and the prescribed forms grows with time.

The same comparison of true and horizon-matched $\dot{\psi}$ is given in Fig. 19 and 20, this time for infall from $r_{0} / 2 M=$ 1.5 $(2 M)$. In all the comparisons of $\dot{\psi}$, the horizon matched choice was made for the prescribed data. The specific 
choice, however, makes little difference in the comparisons. In Fig. 21 a comparison of the true $\dot{\psi}$ and two prescriptions is given for infall from $r_{0} / 2 M=1.5$. (Infall from the small radius magnifies differences of evolution and prescription.) The difference between the basic Bowen-York 14 prescription for $\dot{\psi}$, and the horizon matched prescription, is small, while the difference between either of them and the correct data for $\dot{\psi}$ is very large. The horizon-matched prescription is a slightly better approximation than the "basic" one.

Another way of looking at the difference between the true data and prescribed data is to investigate the effect the difference has on outgoing radiation. Figures Fig. 22, 23, 24 show the waveforms (that is, $\psi(t)$ at large $r$ ) generated by different types of prescribed data. In each case, the solid curve, labeled $t=0.0$, shows the "true" waveform, the waveform generated by evolution of the original, momentarily stationary data at $t=0$. This true waveform is compared with the waveforms evolved from prescribed data placed on later hypersurfaces. The figures show that prescribed data on the $t / 2 M=63.9$ hypersurface leads to an outgoing waveform in reasonably good agreement with the true waveform. For prescribed data on $t / 2 M=93.6$ (with the particle at $r_{p}^{*} / 2 M=3.91$, or $r_{p} / 2 M=3.15$ ), however, large differences are evident between waveform and the true waveform. The disagreement is less severe when the evolved data for $\dot{\psi}$ is retained.

An analogous comparison of waveforms is shown in Fig. 25 for infall from $r_{0} / 2 M=1.5$. Here the effect of prescribed data on the phase of the wave is much more evident than in the $r_{0} / 2 M=15$ case.

In the work of Abrahams and Cook 33, one of the motivations for the present work, the "close limit" was used to evolve prescribed data. This technique [12,15] is applicable to a hypersurface at a time late enough that the colliding bodies can be considered to be inside a single nearly spherical horizon. In this technique one uses only the large- $r$ parts of the initial data of the colliding bodies. This technique was found to be surprisingly successful in dealing with head on collisions of equal mass holes. We saw in Paper I, however, that for very unequal mass holes the close limit is valid only at extremely small separations. For comparison, we have applied the close limit to the present problem. On a sequence of hypersurfaces we replace the hypersurface data by the large- $r$ form of the data, and evolve the results. The resulting energies are shown in Fig. 26. These results, like those in Paper I, show that the close-limit calculations of energy are of limited usefulness.

\section{DISCUSSION AND CONCLUSIONS}

The numerical results of the previous section give a range of comparisons between astrophysically evolved, and "prescribed" hypersurface data (for quadrupole modes.) The first lesson to be learned from these results is that for the particle limit the standard prescriptions for hypersurface data are not adequate for describing an astrophysical strong gravitational field. For a particle falling from $r_{0}=15(2 M)$, for example, an approximation for the radiation using prescribed data on a late hypersurface seriously overestimates the radiated energy. Figure 6 shows that prescribed data for the particle at $r_{p}=3.6 M$ (equivalent to $r_{p}^{*} / 2 M=1.6$ ) gives a radiated energy too large by a factor of 10. Even for a hypersurface with the particle in the marginally strong field at $r_{p}=5.7 M$ (equivalent to $r_{p}^{*} / 2 M=3.4$ ) the energy is overestimated by a factor of two.

This is in marked contrast to the results of Abrahams and Cook [3] for the head on collision of equal mass holes. They prescribed standard data on a late time hypersurface and found predictions of energy in excellent agreement with predictions from numerical relativity. Baker and Li [4], looking further into this problem, found that the predicted energy was remarkably insensitive to the choice of hypersurface. Good predictions could be made with data specified on hypersurfaces over a fairly wide range of times. There are certain technical differences between the nature of the prescribed data we use here, and that used by Abrahams and Cook. In particular, they used fully symmetrized data, whereas our preferred choice of prescribed data is data that is conformally flat, longitudinal data that is frozen and matched at the horizon. This is surely not the origin of the different conclusions. For one thing switching from symmetrized hypersurface data to antisymmetrized, in numerical relativity computations, makes only a minor difference in the results. More important, the work of Baker and Li does not use symmetrized data, and the study by Baker et al. 19] finds excellent results when symmetrized data is replaced by unsymmetrized approximate data. As a further check of this point, we have redone several of our computations using symmetrized prescribed data and, as expected, found only minor differences from the results for other prescriptions.

The crucial difference between the failure of prescribed data here, and its success in the previous studies, must lie in the difference between the head on collision of equal mass throats, and of a large mass throat with a small mass ("particle") one. It is not difficult to see why this should be. It is the same reason the "close limit" approximation is not successful in the collision of very unequal masses. (See Fig. 26 and Paper I.) For the collision of equal masses, a common horizon engulfs both colliding throats as they begin to get into each other's strong field influence. The generation of the large amplitude gravitational waves (i.e., the excitation of quasinormal ringing) more-or-less coincides with the disappearance of the individual throats inside a single connected horizon. For a hypersurface corresponding 
to both throats inside a single horizon, it is plausible (and is found to be valid by the success of the calculations) that details of the geometry near the throats is not important since they cannot influence the region exterior to the horizon. For a collision of high and low mass throats the situation is very different. Strong radiation is generated when the particle is near a coordinate distance $r=1.5(2 M)$, near the peak of the Zerilli potential. For our $t=$ constant slicing this corresponds to a hypersurface for which there is no common horizon, i.e., the particle is still well outside the horizon of the background hole. For this slicing, in fact, the particle always stays outside the background horizon, and the details of its local field are always in causal connection with infinity. The fault would not seem to be in the slicing. A $t=$ constant slicing was the choice in the method used by Abrahams and Cook [3] and by Baker and Li [4, and is an obviously natural slicing for the particle infall problem.

We conclude from these considerations that the fault lies in the nature of the prescribed data we have been using, i.e., the standard choice of conformally flat longitudinal hypersurface data. As shown in the figures of the previous section, there is a dramatic difference between the true hypersurface data on a late hypersurface, and prescribed data. There are two strong reasons to suspect that the most important difference between true hypersurface data and any of the prescribed data is the difference in $\dot{\psi}$. First, our argument above suggests that the failure of prescribed data in the particle limit lies in the failure to describe fields near the particle. In Figs. 17 - 21, we see that there is (at least visually) a large difference between the true and the prescribed data close to the particle. This difference can be ascribed to the constraints on conformally flat longitudinal data. The sharp variations in $\dot{\psi}\left(r^{*}\right)$ are, intuitively, features that should couple strongly to radiation. The inability of the prescribed data to model these features, must be viewed as potentially important. The second reason for focusing attention on $\dot{\psi}$ is that the hypersurface data for $\dot{\psi}$ appears to be much more important than that for $\psi$. This was the conclusion in the study by Baker et al. [19]. In that work, which used colliding throats, and no stress energy, Einstein's equations were linearized in the momentum of the throats and a clear identification could be made of how much of the radiated energy could be ascribed to the initial 3-geometry, how much to the momentum, i.e., the initial extrinsic curvature, and how much to the interaction of the two. It was found that except for extremely small initial momentum the radiation was almost completely due to the extrinsic curvature. This was called "momentum dominance" by Baker et al. Here we cannot make such a clear distinction. Due to the moving particle source, treated as a stress energy contribution, we cannot ascribe the radiation to the two kinds of initial data information. Nevertheless, the energy results in our Figs. -6 show that some other form of "momentum dominance" applies to the particle infall; the energy radiated is much more sensitive to the details of the extrinsic curvature than to the details of the 3-geometry.

The insights provided by the particle limit have helped to clarify what is needed in the way of hypersurface data for numerical evolution. A direct consequence of the present study is the realization that prescribed data will not work as successfully for unequal mass head on collisions as for the equal mass case. At some ratio of masses of the colliding holes the use of prescribed data on a late time hypersurface will start to give a significant overestimate of the radiated energy. We have, so far, studied only the quadrupole perturbations. Higher multipole moments are less important astrophysically, and are not likely to lead to very different conclusions.

The head on collision, of course, is neither astrophysically plausible, nor an interesting source of outgoing radiation. The interesting case is the last stage in the orbital decay of a binary pair of holes. For this one would like to start with astrophysically reasonable data on a "as late as possible" hypersurface. An understanding of how to do this for the head on case is a necessary step (and perhaps a sufficient one) towards an understanding of the more general problem. With that motivation, we shall, in a subsequent paper, investigate what can be done to provide good late time data. We shall, in particular, abandon the traditional choice of conformally flat, longitudinal data, and, instead, shall focus on data that gives a good description of the field near the particle.

[1] A. A. Abramovici et al., Science 256, 325 (1992); K. S. Thorne, submitted to Proceedings of Snowmass 94 Summer Study on Particle and Nuclear Astrophysics and Cosmology, eds. W. W. Kolb and R. Peccei (World Scientific, Singapore).

[2] Proceedings of the November 1994 meeting of the Grand Challenge Alliance to study black hole collisions may be obtained by contacting E. Seidel at NCSA (unpublished).

[3] A. M. Abrahams and G. B. Cook, Phys. Rev. D50, R2364 (1994).

[4] J. Baker and C. B. Li, Class. Q. Grav. 14, L77 (1997).

[5] C. O. Lousto and R. H. Price, Phys. Rev. D55, 2124 (1997). (Paper I)

[6] V. Moncrief, Ann. Phys. (NY) 88, 323 (1974).

[7] T. Regge and J. A. Wheeler, Phys. Rev. 108, 1063 (1957).

[8] F. J. Zerilli, Phys. Rev. Lett. 24737 (1970). 
[9] For example, J. W. York, Jr. and T. Piran, Spacetime and Geometry, edited by R. Matzner and L. Shepley (Univ. of Texas Press, Austin, 1982), pp. 147 - 176, and references therein.

[10] D. R. Brill and R. W. Lindquist, Phys. Rev. 131, 471 (1964).

[11] C. W. Misner, Ann. of Phys. 24, 102 (1963); R. W. Lindquist, J. Math. Phys. 4, 938 (1963).

[12] R. H. Price and J. Pullin, Phys. Rev. Lett. 72, 3297 (1994).

[13] F. J. Zerilli, Phys. Rev. D2 2141 (1970).

[14] J. M. Bowen and J. W. York, Jr., Phys. Rev. D21, 2047 (1980).

[15] A. Abrahams and R. H. Price, Phys. Rev. D53, 1963 (1996)

[16] J.F. Bowen, Gen. Rel. and Grav., 11, 227 (1979).

[17] G. B. Cook, Phys. Rev. D44, 2983 (1991).

[18] J. W. York, Jr., J. Math. Phys. 14, 456 (1973).

[19] J. Baker, A. Abrahams, P. Anninos, S. Brandt, R. Price, J. Pullin and E. Seidel, Phys. Rev. D55, 829 (1997).

FIG. 1. Geometry in the conformally related flat space.

FIG. 2. A cell of the computational grid containing a segment of the particle worldline. Grid nodes are shown coordinatized with $t, r^{*}$ labels, and with values of the null coordinates $u \equiv t-r^{*}$ and $v \equiv t+r^{*}$. The areas $A_{1} \cdots A_{4}$ are used as weights in the numerical algorithm.

FIG. 3. Hypersurfaces of constant $t$ in the Schwarzschild spacetime. The trajectory is shown of a particle starting from rest at position $r_{0} / 2 M=15$, and falling inward on a radial geodesic. Also shown are hypersurfaces of constant time at $t / 2 M=20,40,60$ and 80 . The hypersurfaces are labeled with the value of $t$, of the particle position $r_{p}$, and of momentum parameter $P$. The $t / 2 M=20$ hypersurface, for example, can also be referred to as the $P / m_{0}=0.05$ hypersurface, the $r_{p} / 2 M=14.6$ hypersurface, or the $r_{p}^{*} / 2 M=17.2$ hypersurface.

FIG. 4. Radiated Energy, for infall from $r_{0}=1.5(2 M)$ Energy is shown as a function of the $r_{p}^{*}$ label of a hypersurface and results are given for several types of prescribed hypersurface data. Radiated energy computed is always the energy radiated starting from the time of the hypersurface on which data is prescribed. See text for details.

FIG. 5. Radiated Energy, for infall from $r_{0}=1.5(2 M)$. Energy is shown as a function of the $r_{p}^{*}$ label of a hypersurface. On that hypersurface the true, i.e., evolved, data for $\dot{\psi}$ has been retained, but frozen prescribed data for $\psi$ has been substituted for the true $\psi$.

FIG. 6. Radiated Energy, for infall from $r_{0}=15(2 M)$. Energy is shown as a function of the $r_{p}^{*}$ label of a hypersurface and results are given for several types of prescribed hypersurface data. The computed energy is always the energy radiated starting from the time of the hypersurface on which data is prescribed. See text for details.

FIG. 7. The true quadrupole $\psi\left(r^{*}\right)$ for infall from $r_{0}=15(2 M)$, i.e., $\psi$ computed by numerical evolution from the initial hypersurface. Curves are shown for several hypersurfaces labeled with $r_{p}^{*}$, the value of the particle's $r^{*}$ location on each hypersurface.

FIG. 8. The true quadrupole $\psi\left(r^{*}\right)$ for infall from $r_{0}=15(2 M)$, shown on late hypersurfaces. For $t / 2 M=109.7$ the shape of $\psi$ shows outgoing quasinormal radiation; for $t / 2 M=149.6$ both ingoing and outgoing quasinormal oscillations are evident.

FIG. 9. The comparison of true and prescribed quadrupole $\psi$ for infall from $r_{0}=15(2 M)$. The form of the true data $\psi\left(r^{*}\right)$ for infall from Misner data on the original hypersurface, is compared with the frozen prescribed data on the hypersurface at $t / 2 M=52.2$.

FIG. 10. For infall from $r_{0}=15(2 M)$, the evolved data is compared with the frozen prescribed data, at $t / 2 M=96.9$ 
FIG. 11. For infall from $r_{0}=15(2 M)$, the evolved data is compared with the frozen prescribed data, at $t / 2 M=100.2$.

FIG. 12. Misner prescribed data for infall from $r_{0}=1.5(2 M)$, shown on a sequence of hypersurfaces.

FIG. 13. Frozen prescribed data for infall from $r_{0}=1.5(2 M)$, shown on a sequence of hypersurfaces.

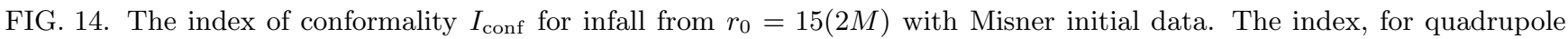
perturbations, is equivalent to $\mathrm{H}_{2}-K$ in the RW gauge, and is a gauge invariant measure of the extent to which the hypersurface 3 -geometry fails to be conformally flat (see text). The index is given, as a function of $r^{*}$, for several different hypersurfaces. The noisy nature of the curves is caused by the need to take second differences of numerical results to compute $I_{\text {conf }}$.

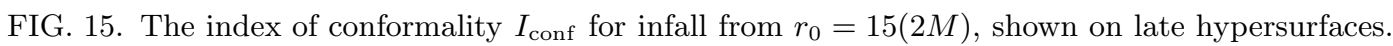

FIG. 16. For $r_{0} / 2 M=15$, the true (i.e., evolved) form of $\dot{\psi}$ compared with the horizon matched prescribed form of $\dot{\psi}$, for $t / 2 M=52.2$.

FIG. 17. For $r_{0} / 2 M=15$, the true (i.e., evolved) form of $\dot{\psi}$ compared with the horizon matched prescribed form of $\dot{\psi}$, for $t / 2 M=96.9$.

FIG. 18. For $r_{0} / 2 M=15$, the true (i.e., evolved) form of $\dot{\psi}$ compared with the horizon matched prescribed form of $\dot{\psi}$, for $t / 2 M=100.2$.

FIG. 19. For $r_{0} / 2 M=1.5$, the true (i.e., evolved) form of $\dot{\psi}$ compared with the horizon matched prescribed form of $\dot{\psi}$, for $r_{p} / 2 M=1.34$.

FIG. 20. For $r_{0} / 2 M=1.5$, the true (i.e., evolved) form of $\dot{\psi}$ compared with the horizon matched prescribed form of $\dot{\psi}$, for $r_{p} / 2 M=1.21$.

FIG. 21. For $r_{0} / 2 M=1.5$, the true, i.e., evolved, form of $\dot{\psi}$ compared with the horizon matched prescribed form of $\dot{\psi}$, and with the Bowen-York form.

FIG. 22. The quadrupole waveform, $\psi(t)$ at $r / 2 M=1000$, for infall from $r_{0} / 2 M=15$. Three curves are shown corresponding to the true waveform (data prescribed at $t / 2 M=0$ ) and to the waveforms generated when frozen $\psi$ data and horizon matched $\dot{\psi}$ data are imposed on later hypersurfaces.

FIG. 23. The quadrupole waveform, $\psi(t)$ at $r / 2 M=1000$, for infall from $r_{0} / 2 M=15$. Three curves are shown corresponding to the true waveform (data prescribed at $t / 2 M=0$ ) and to the waveforms generated when horizon matched $\dot{\psi}$ data are imposed on later hypersurfaces and the true, i.e., evolved, values of $\psi$ are retained.

FIG. 24. The quadrupole waveform, $\psi(t)$ at $r / 2 M=1000$, for infall from $r_{0} / 2 M=15$. Three curves are shown corresponding to the true waveform (prescribed data a $t / 2 M=0$ ) and to the waveforms generated when frozen $\psi$ data are imposed on later hypersurfaces and the true, i.e., evolved, values of $\dot{\psi}$ are retained. 
FIG. 25. The quadrupole waveform, $\psi(t)$ at $r / 2 M=1000$, for infall from $r_{0} / 2 M=1.5$. The solid curve shows the "true" waveform evolved directly from the initial data at $t / 2 M=0$. The dashed curve gives the waveform generated when prescribed data (horizon-frozen data) for $\psi$, and evolved data for $\dot{\psi}$ are placed on a late hypersurface.

FIG. 26. Radiated energy for infall from $r_{0}=15(2 M)$ computed with the close limit. On every hypersurface close limit data was substituted and evolved. The computed energy is plotted as a function of the hypersurface parameter on which the close limit data was substituted. 


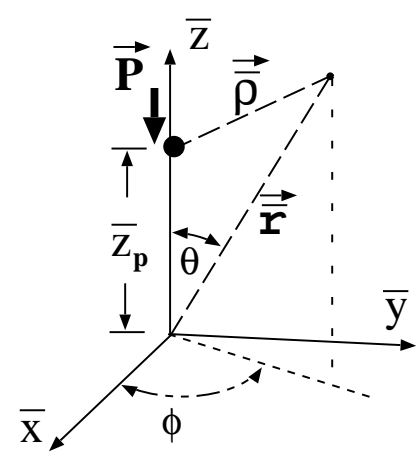




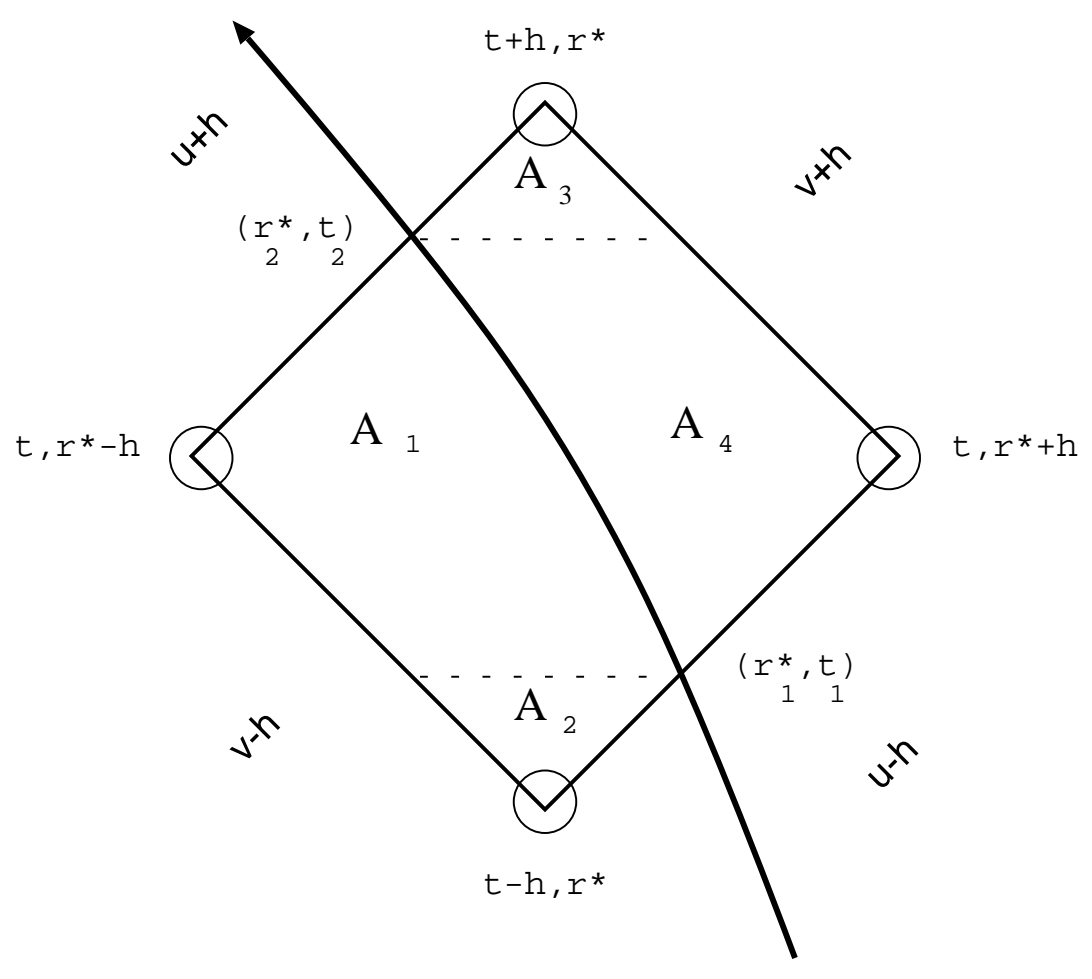




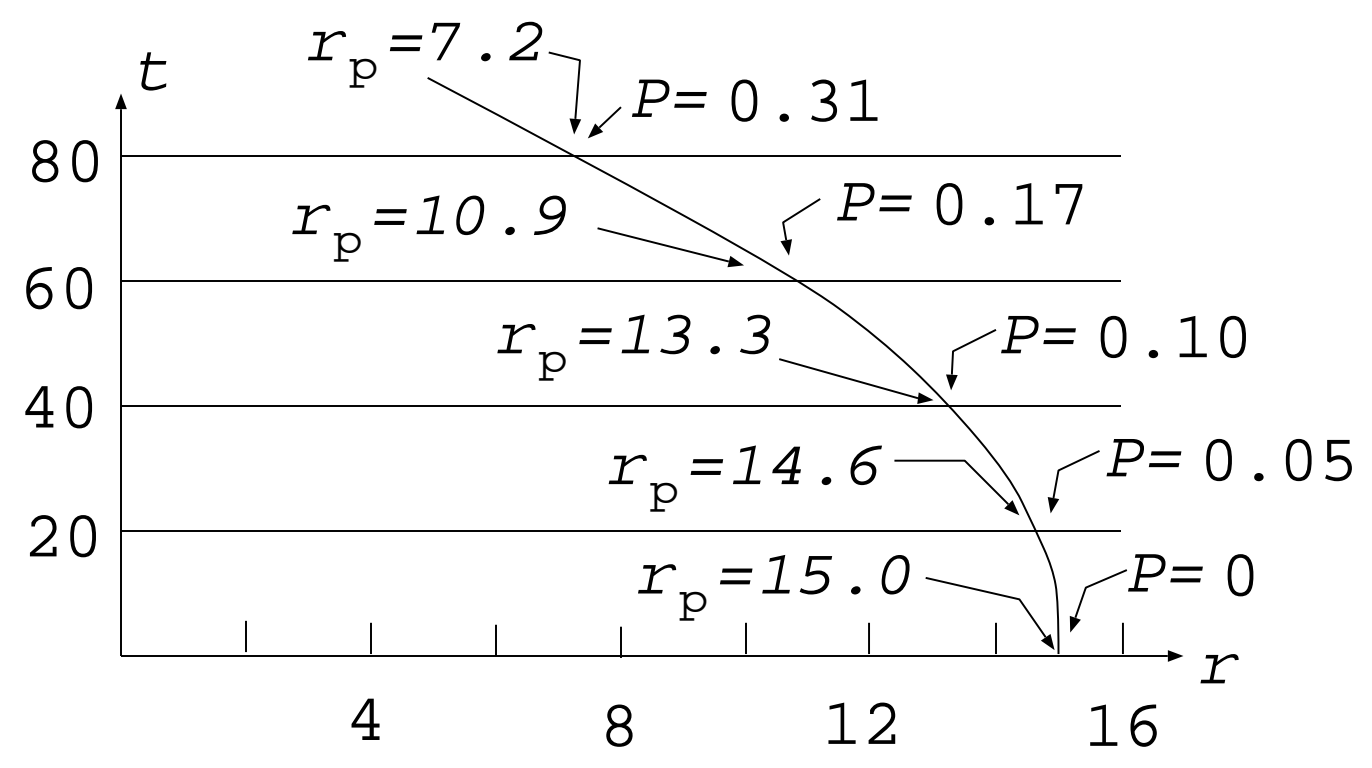




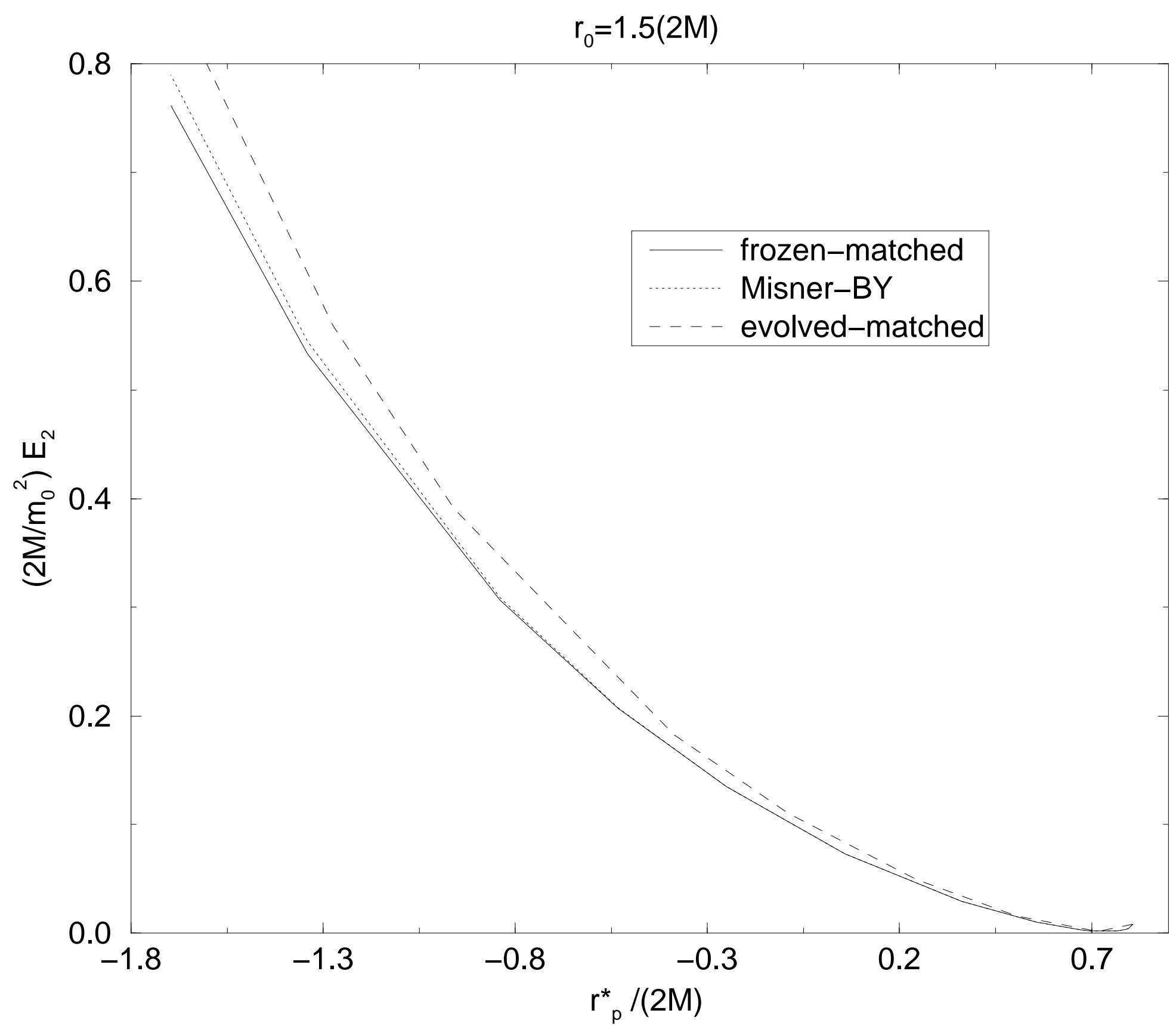




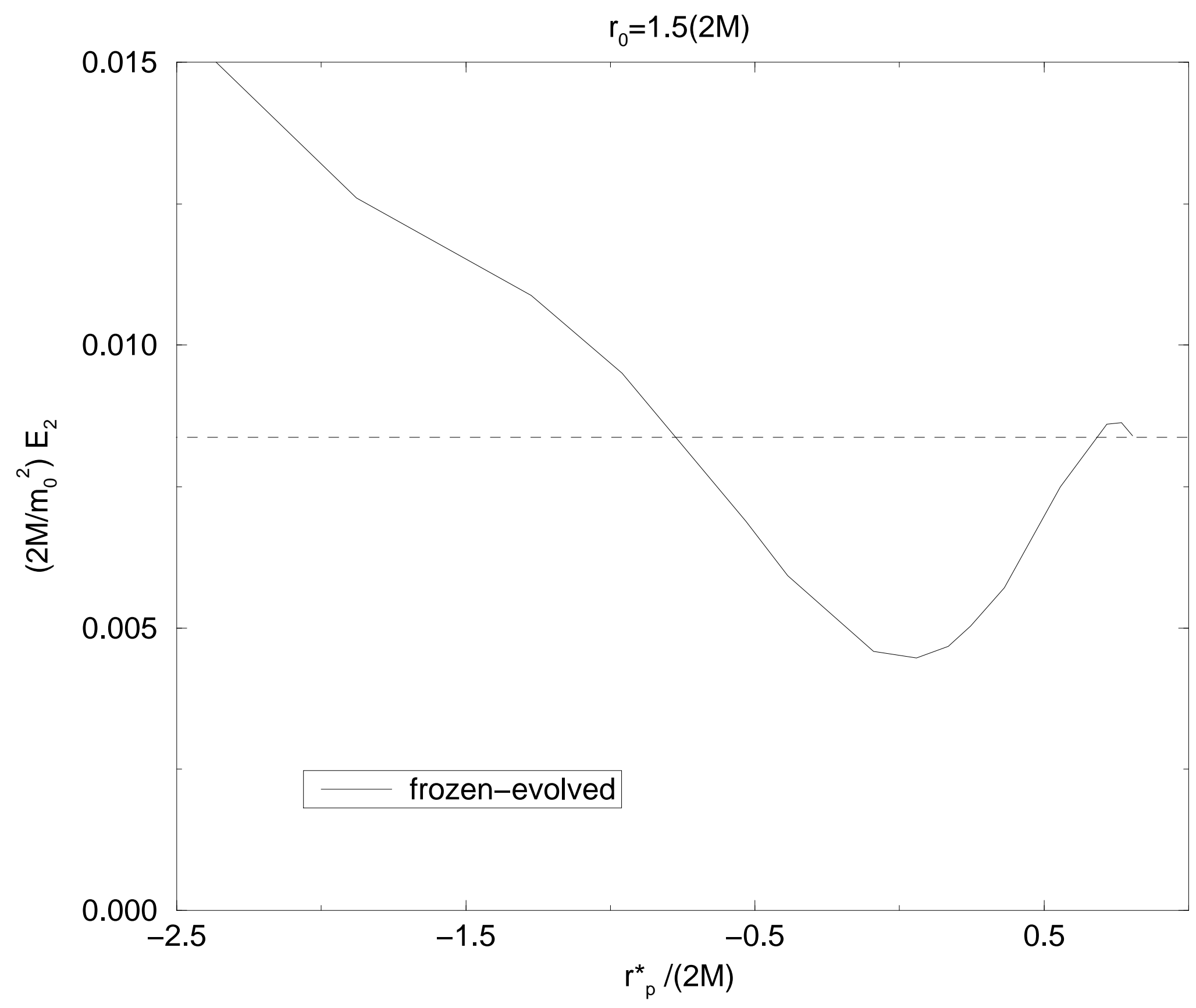


$r_{0}=15(2 M)$

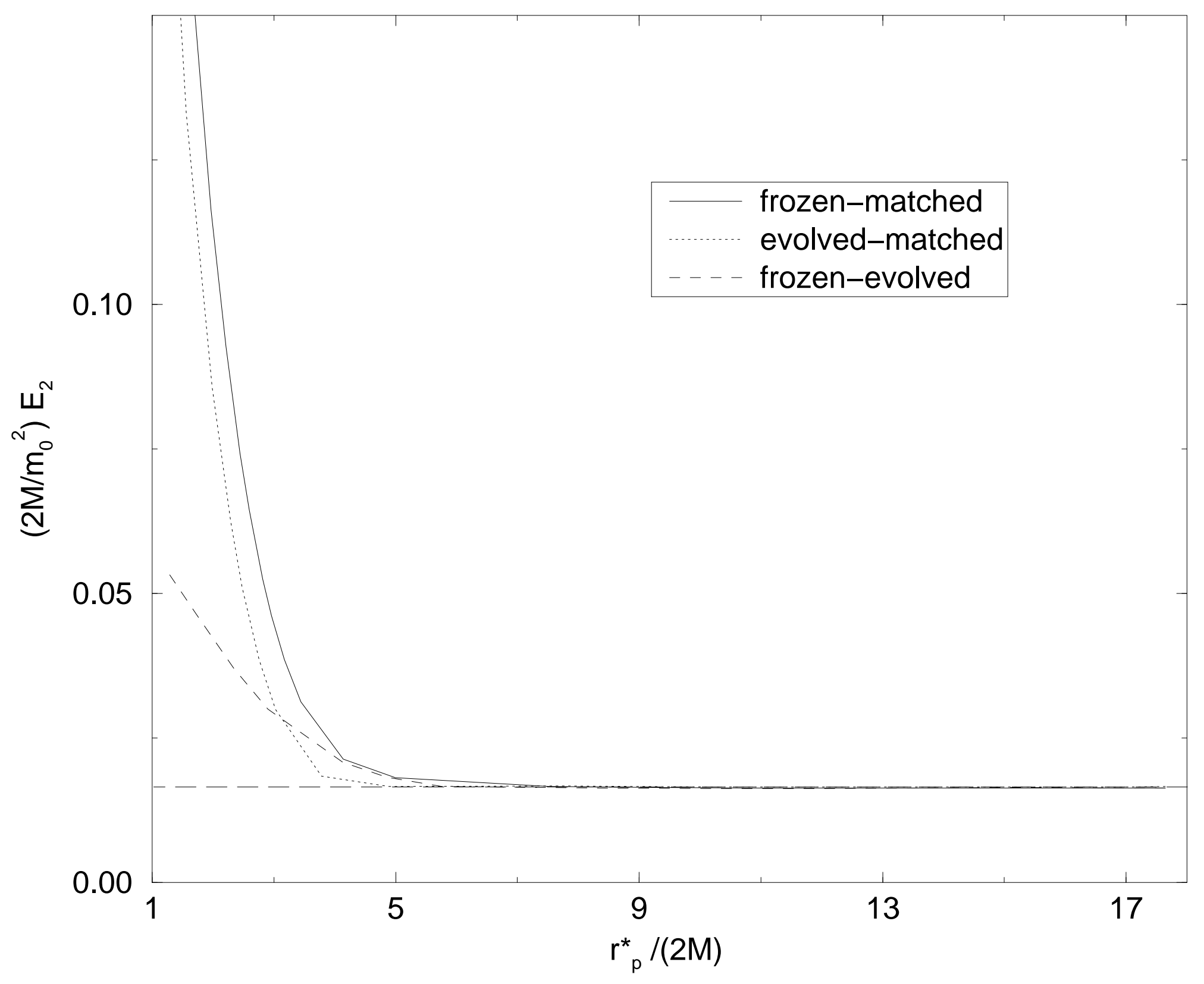




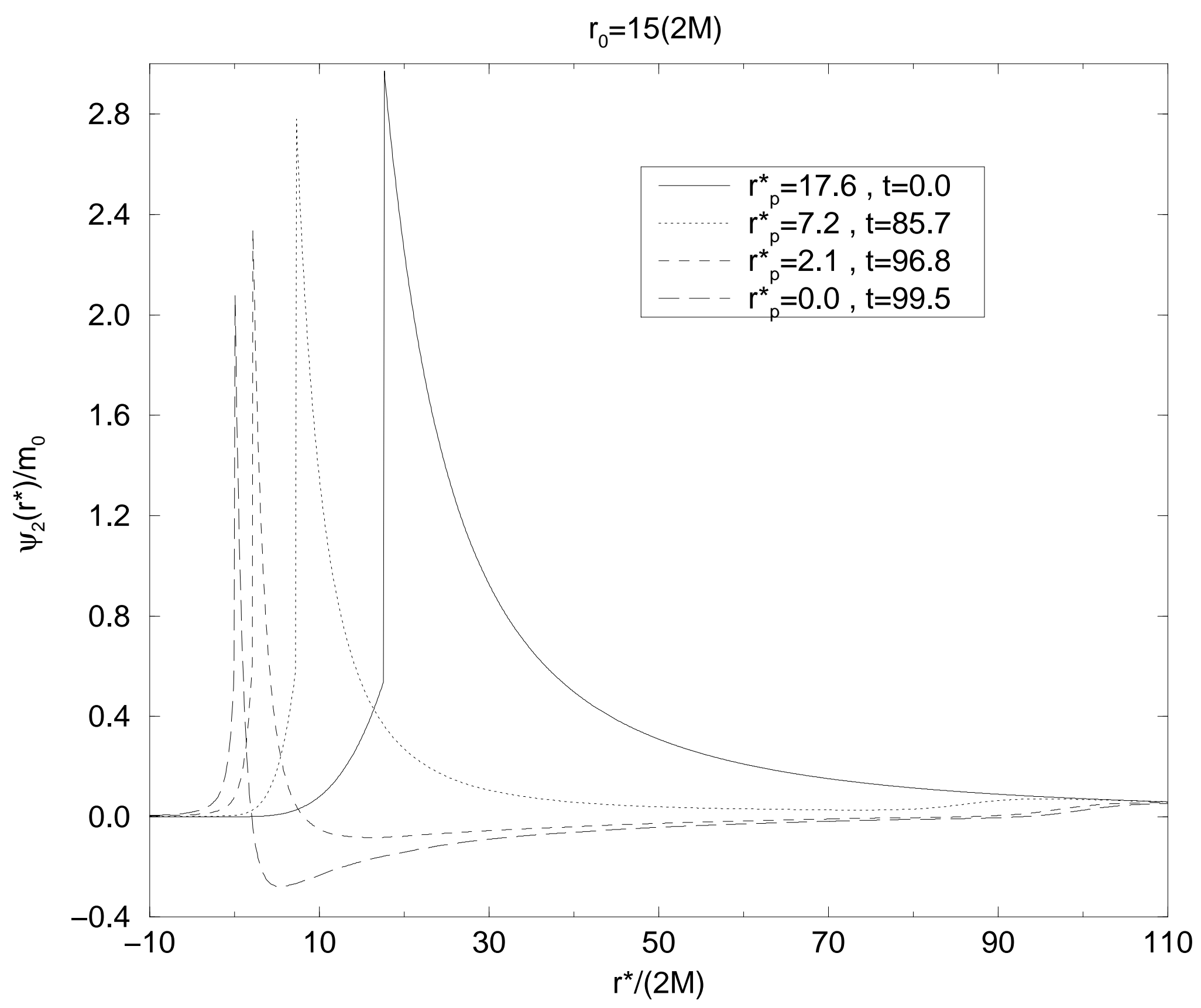




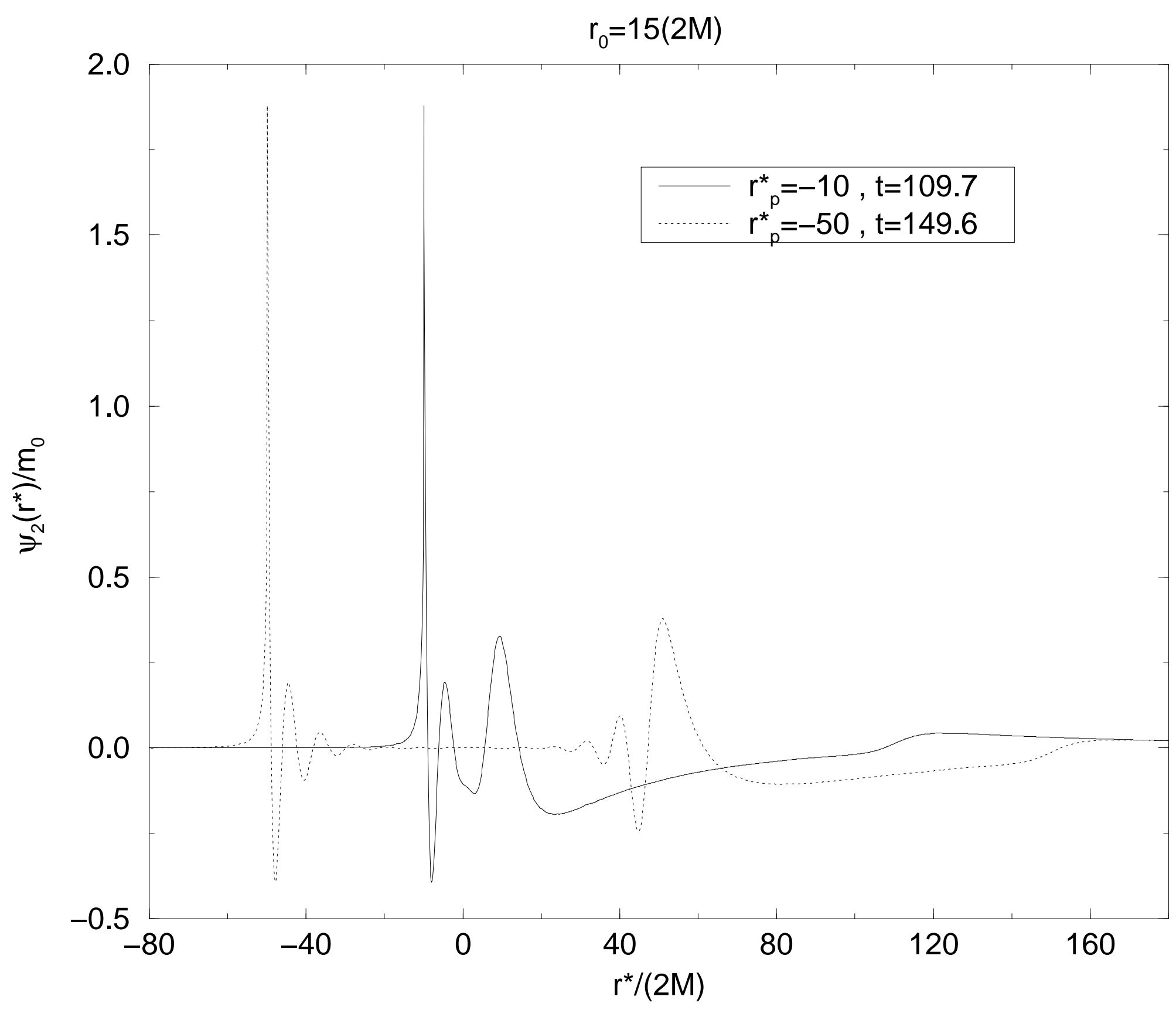




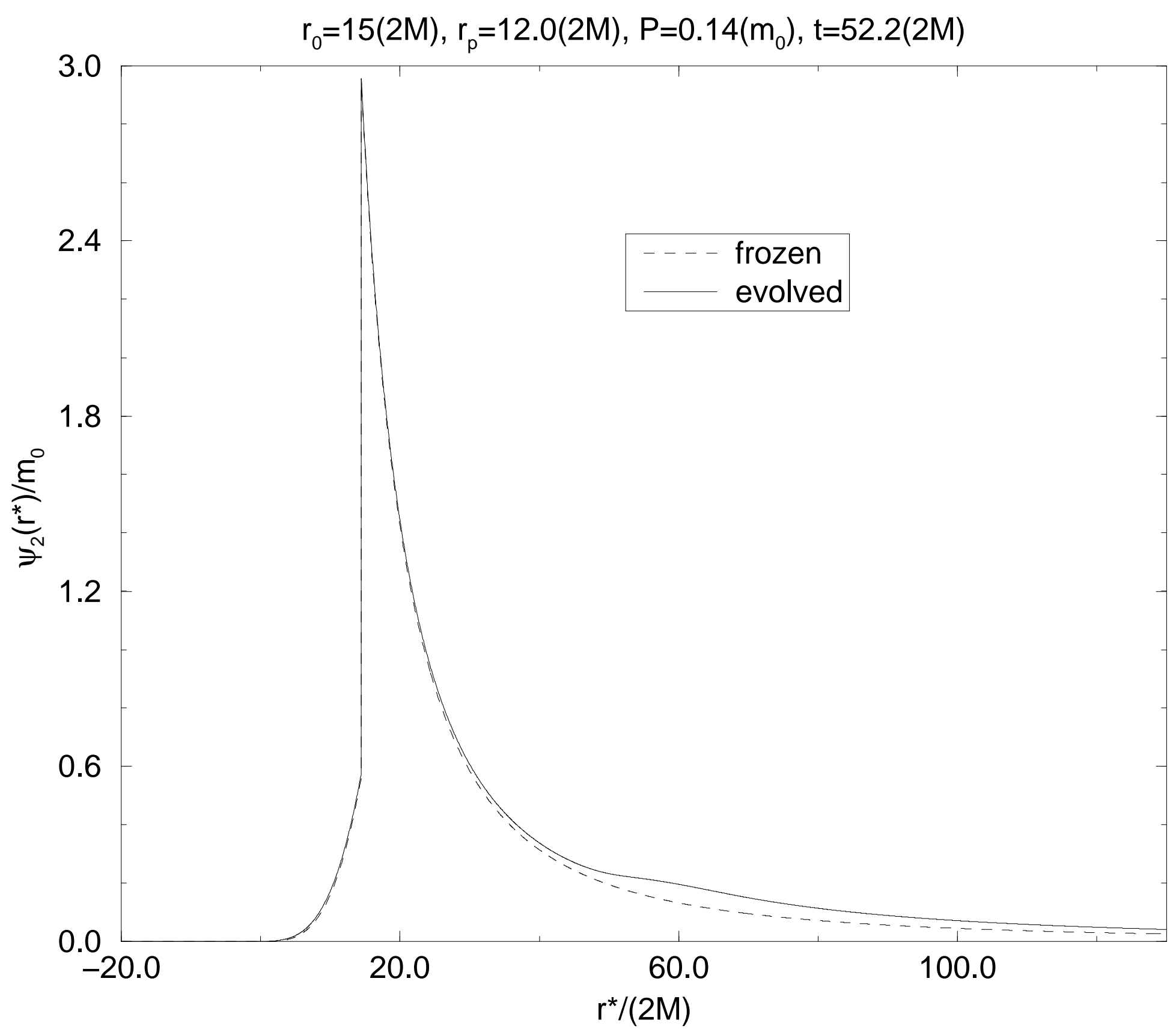




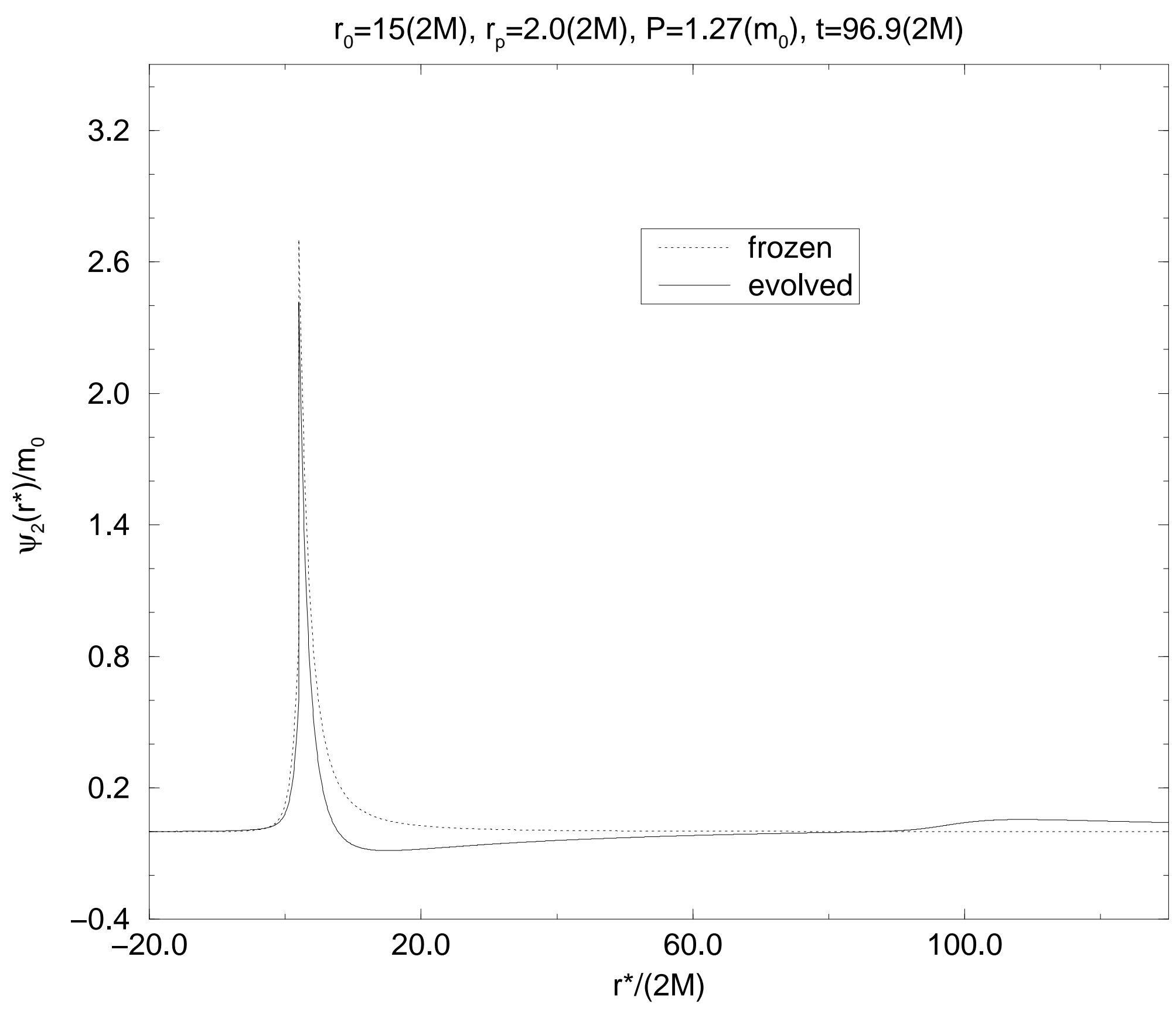




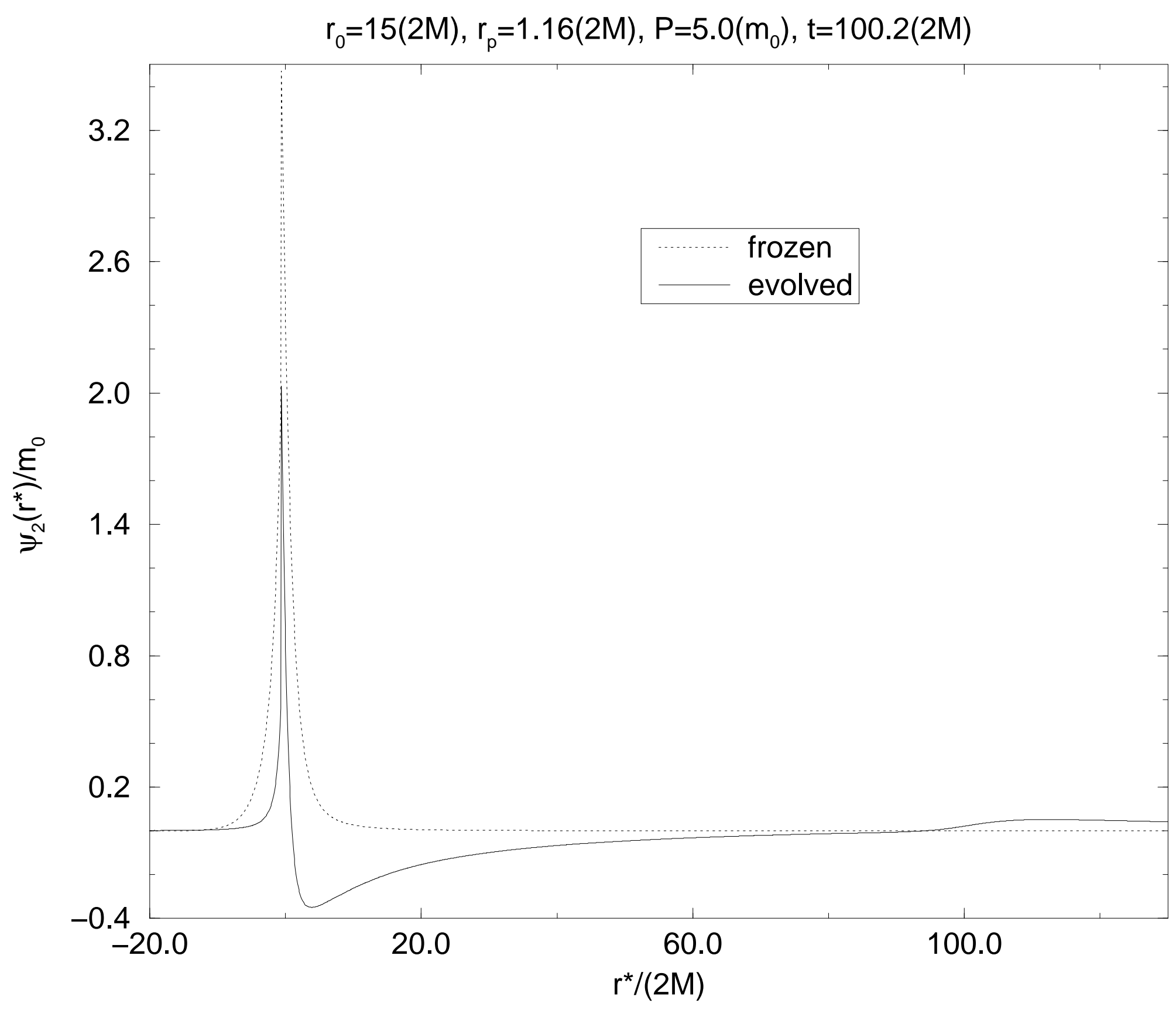




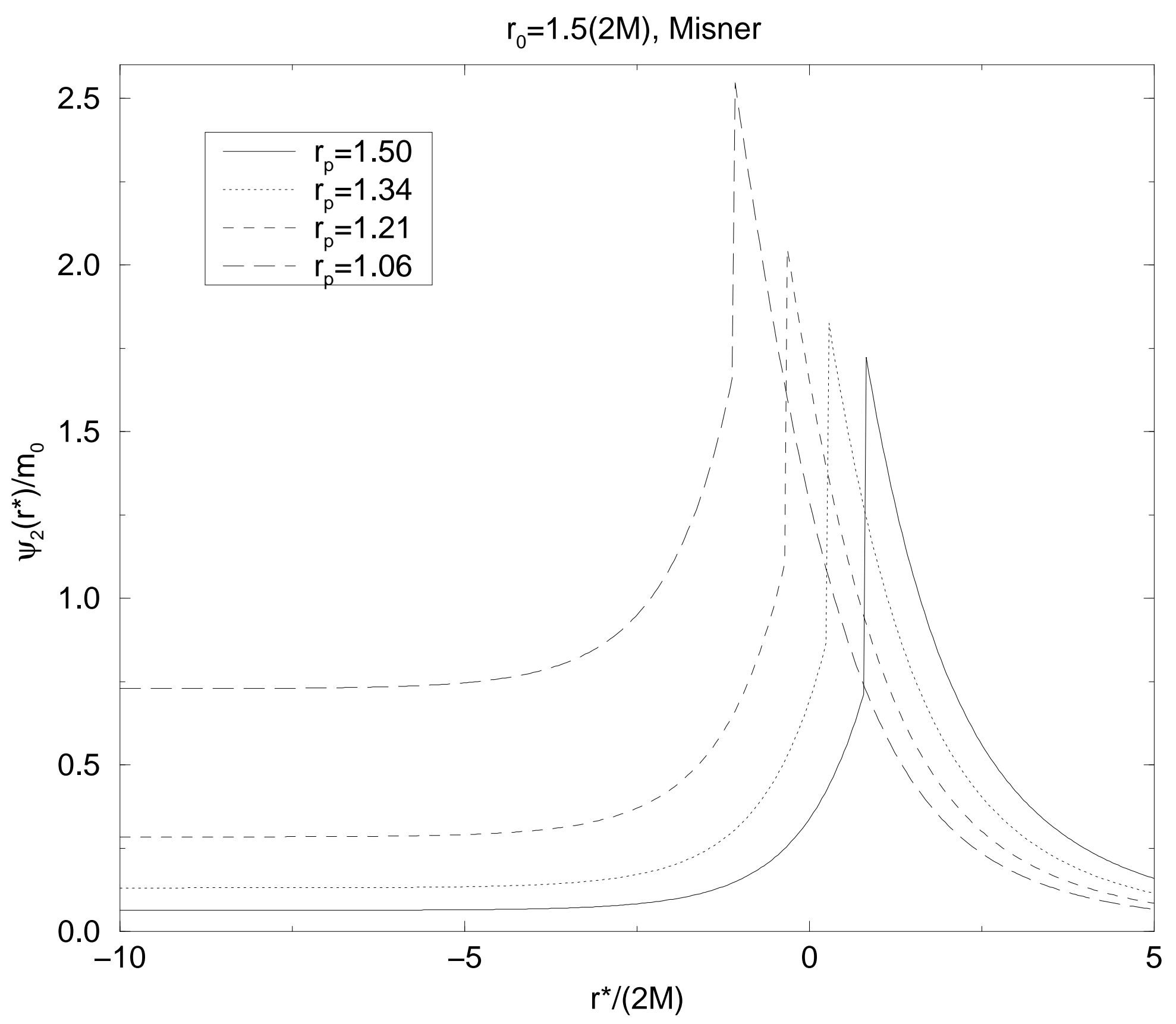




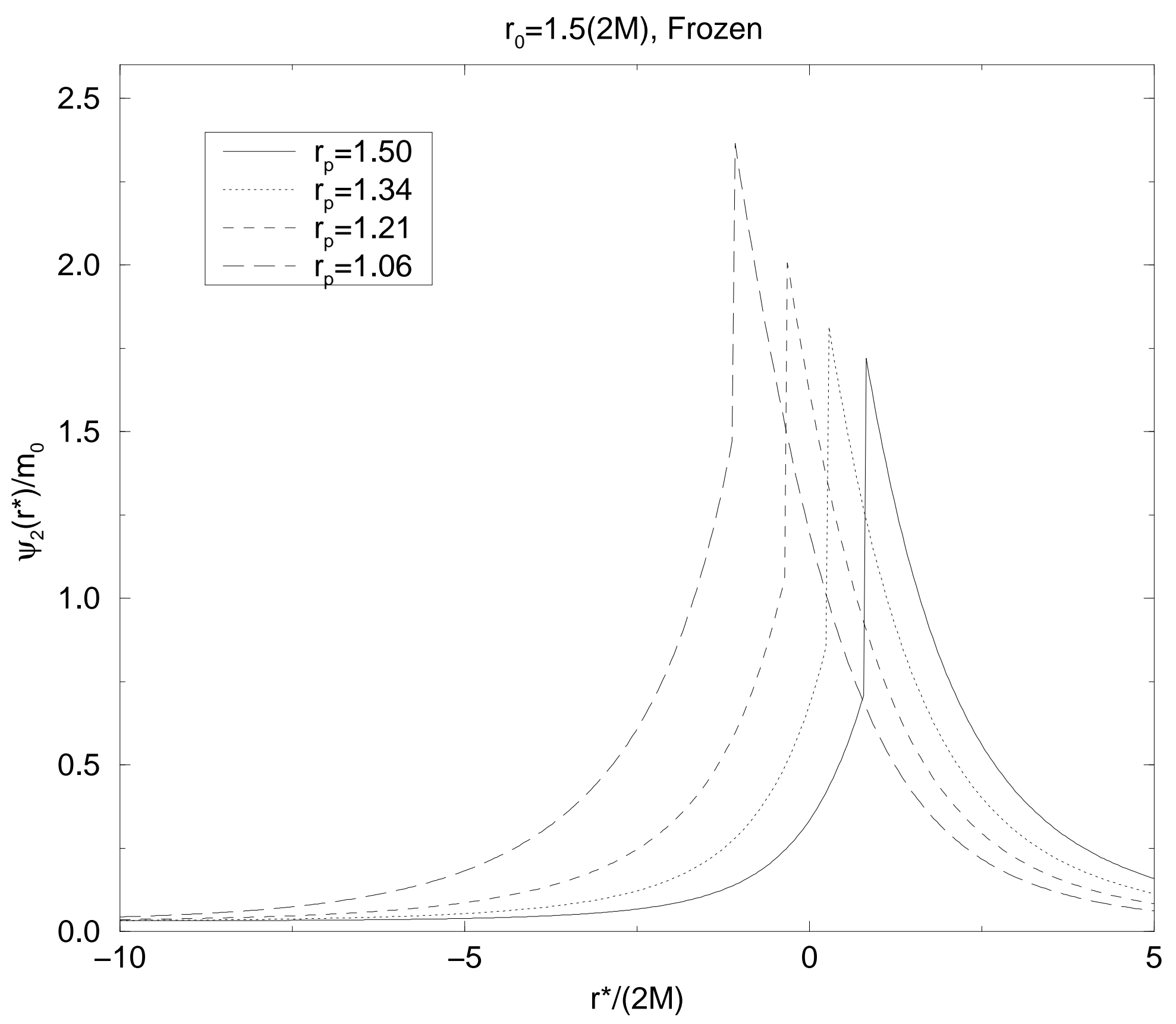




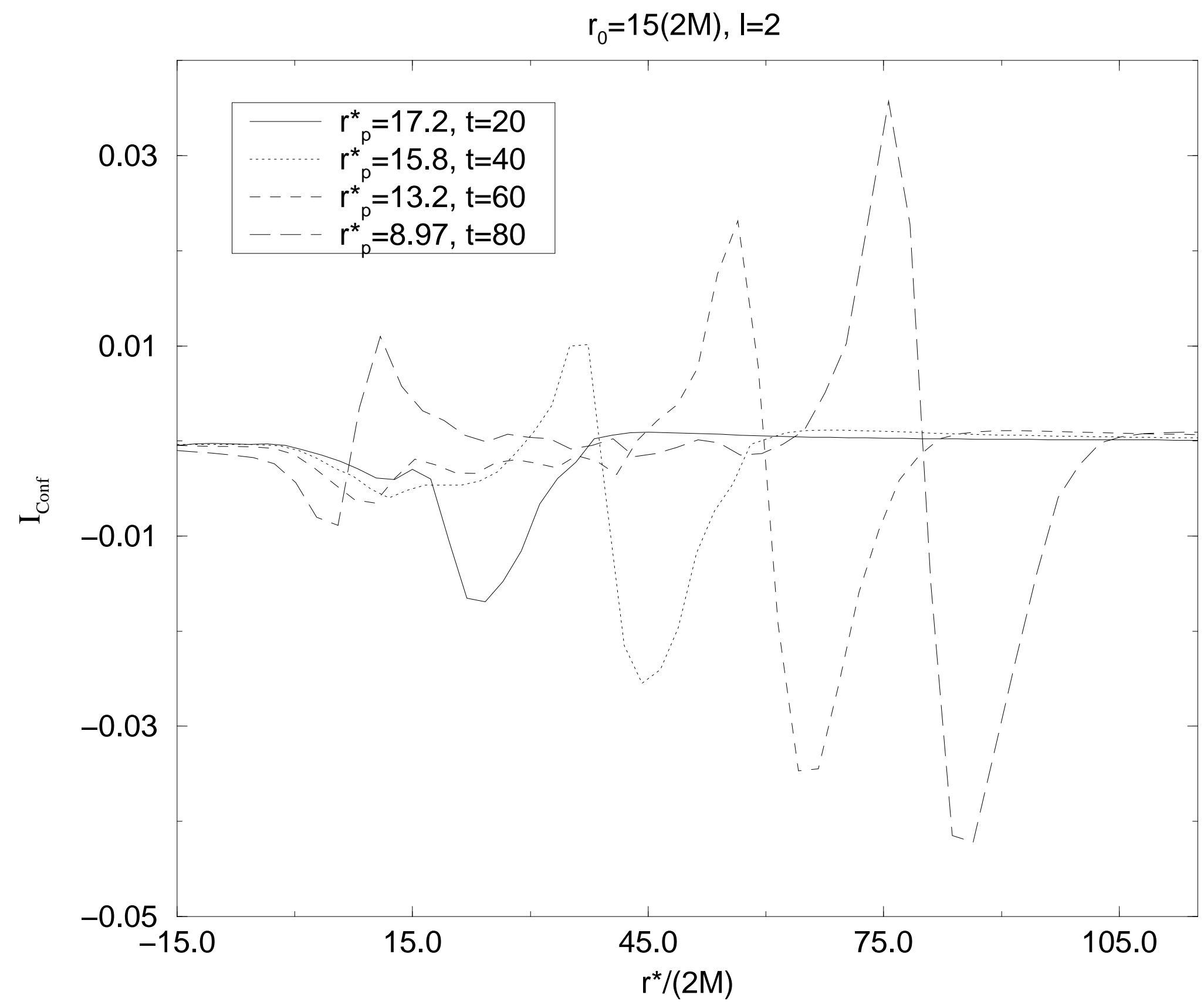




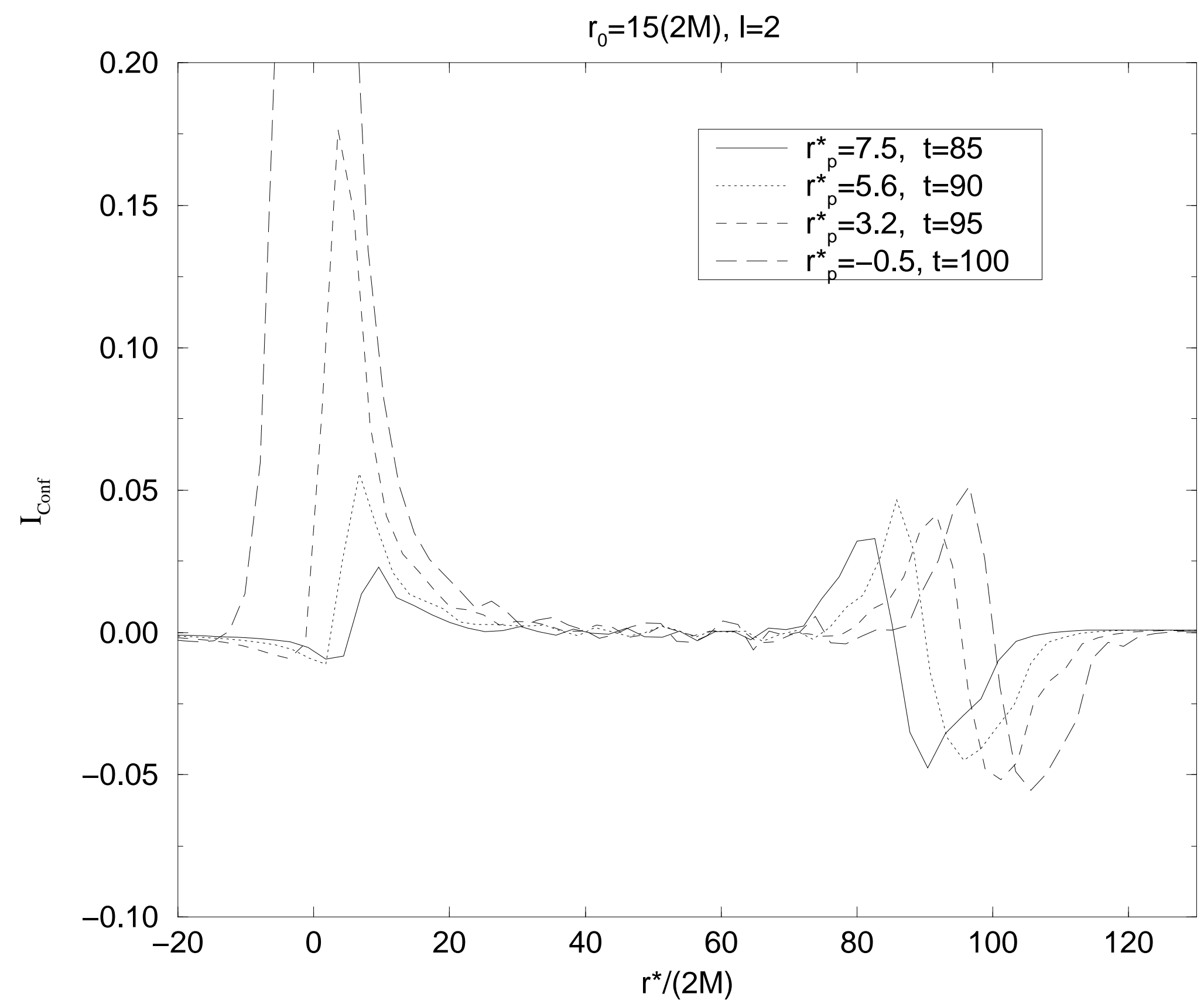




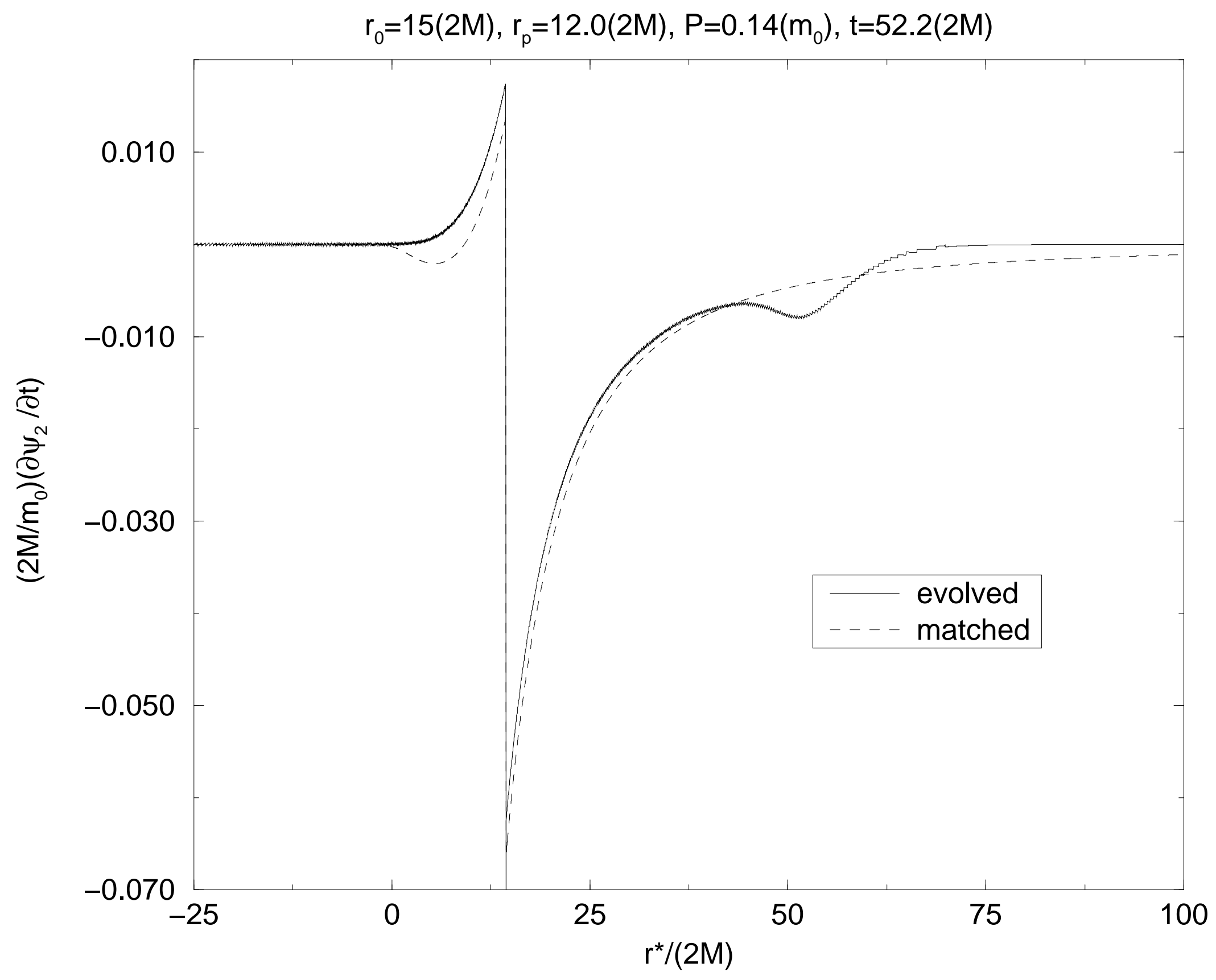




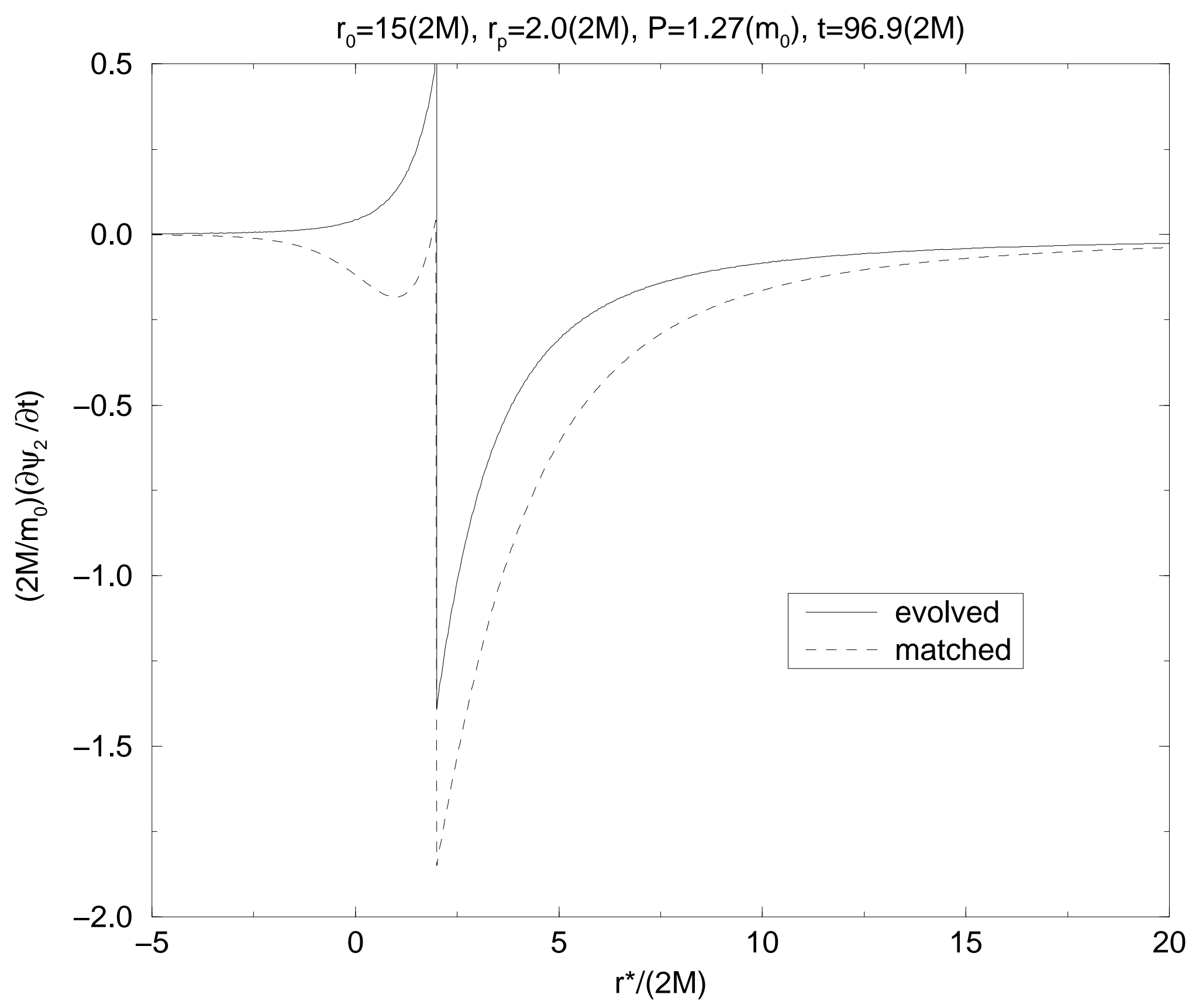




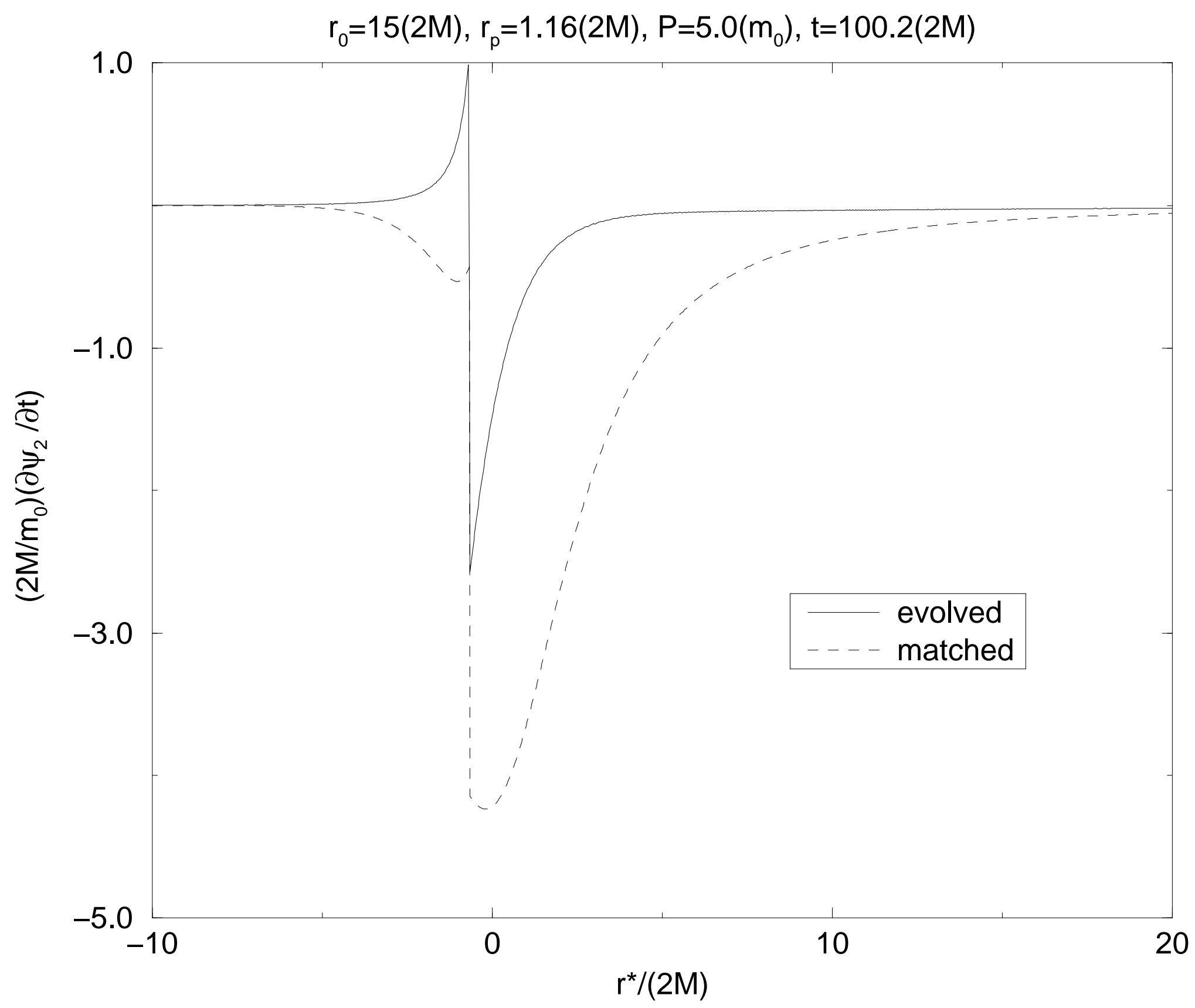




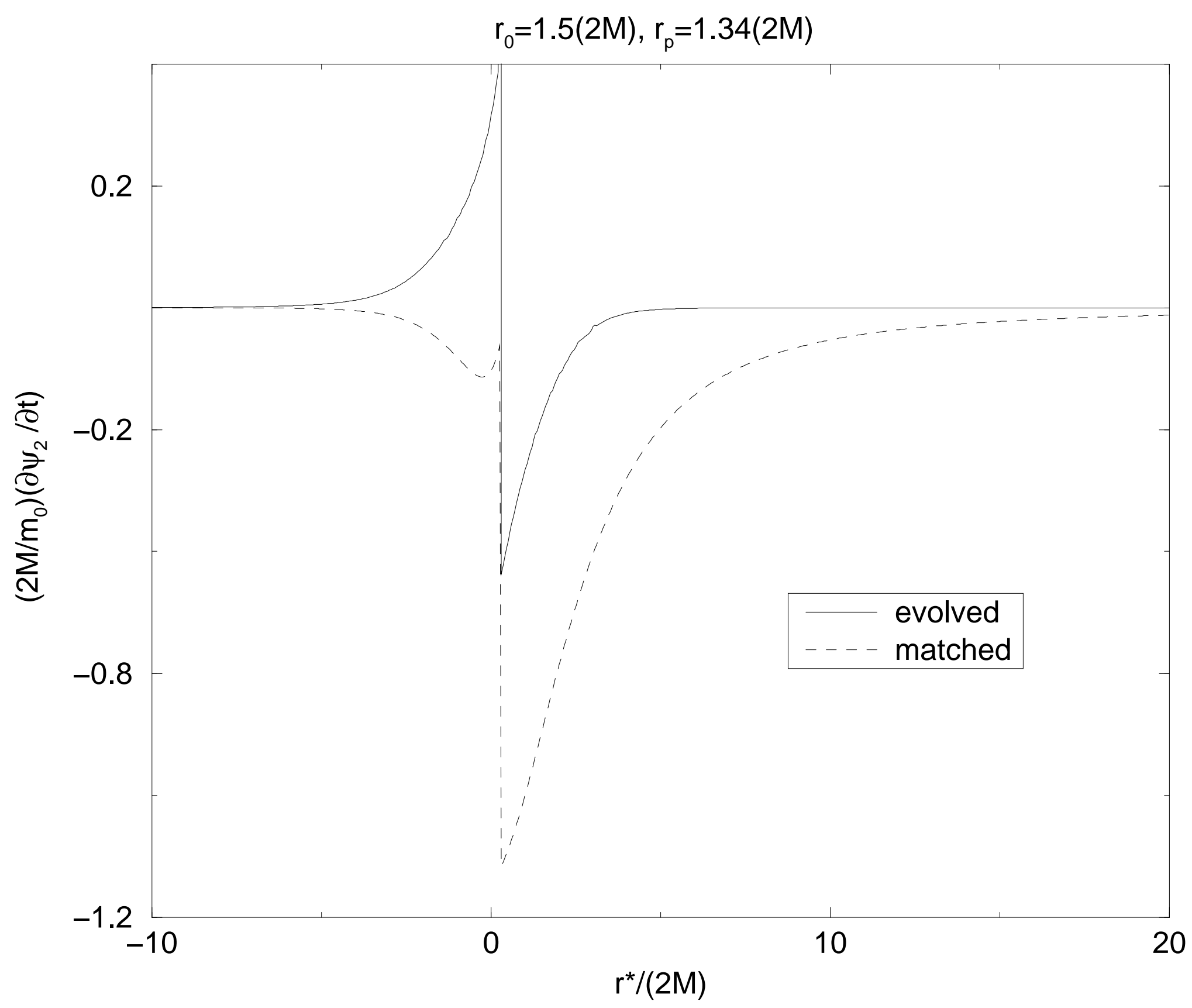




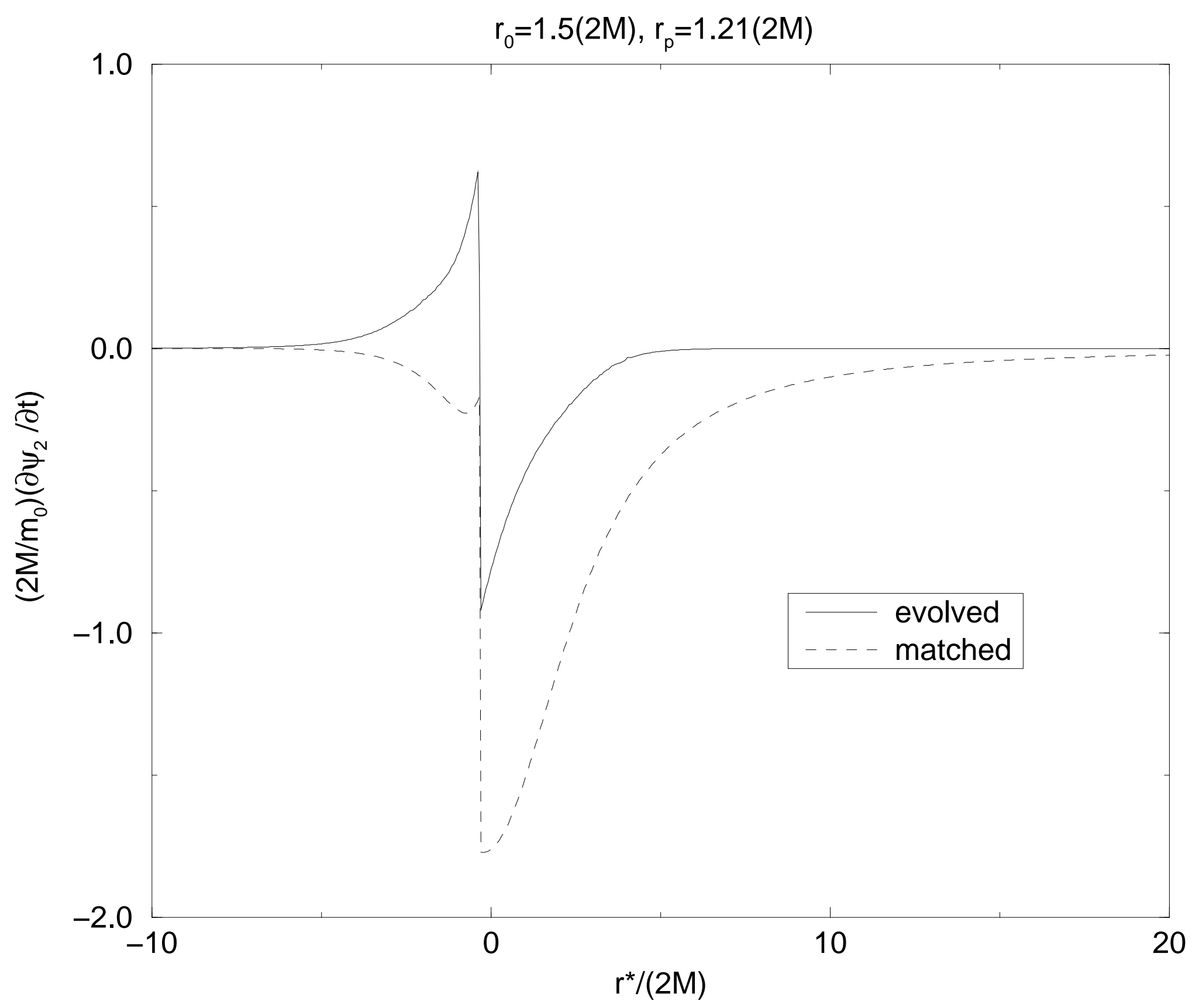




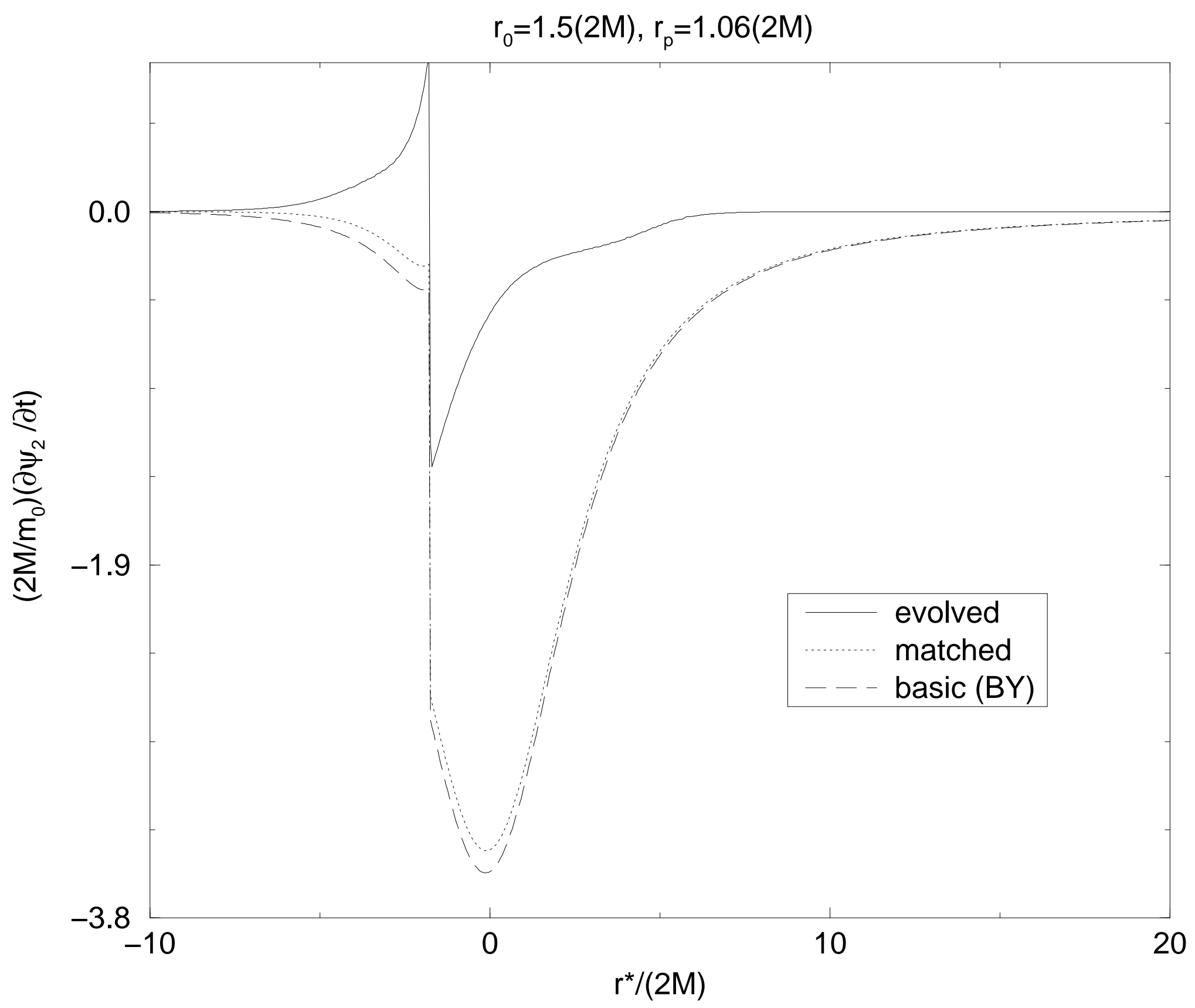




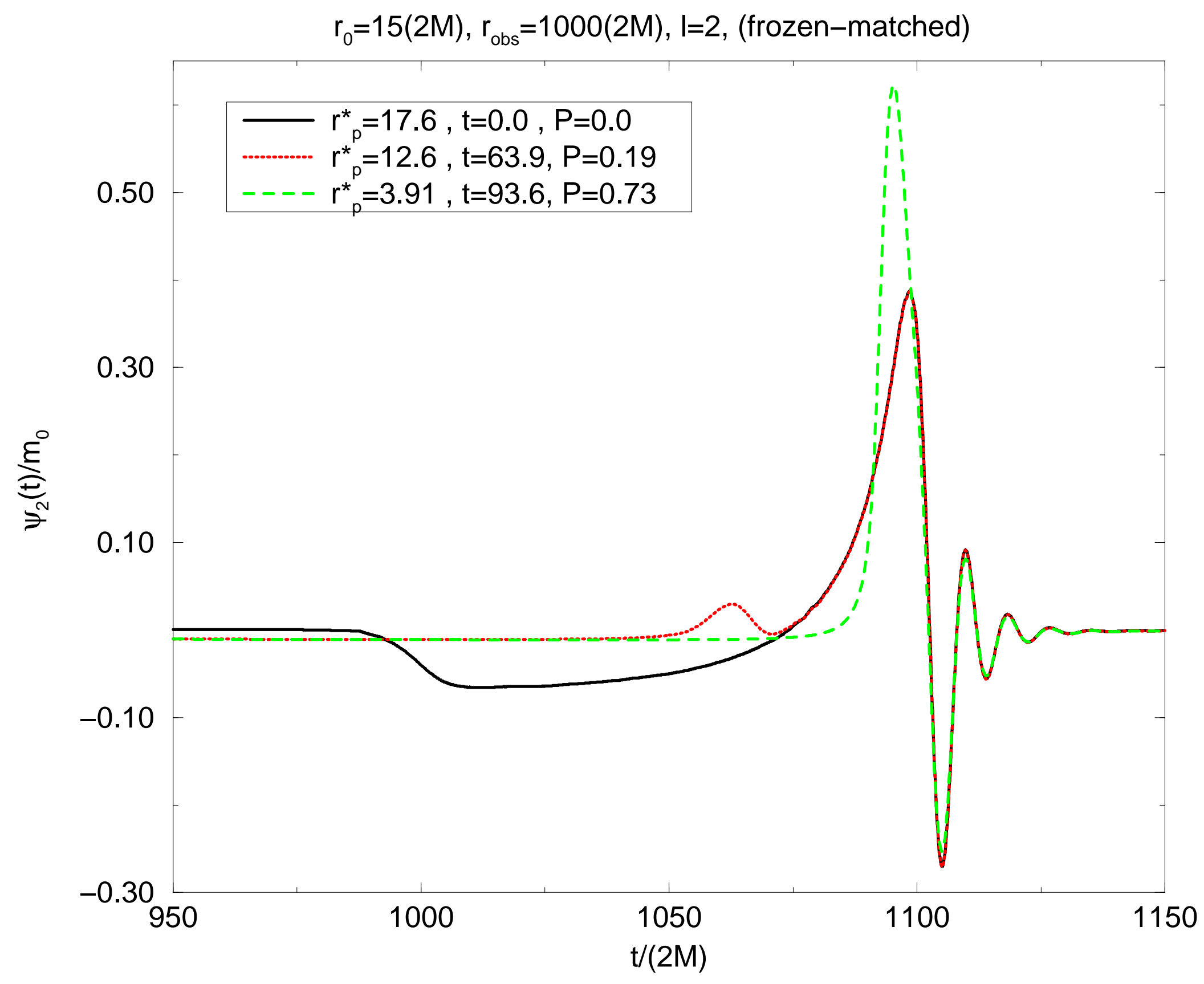




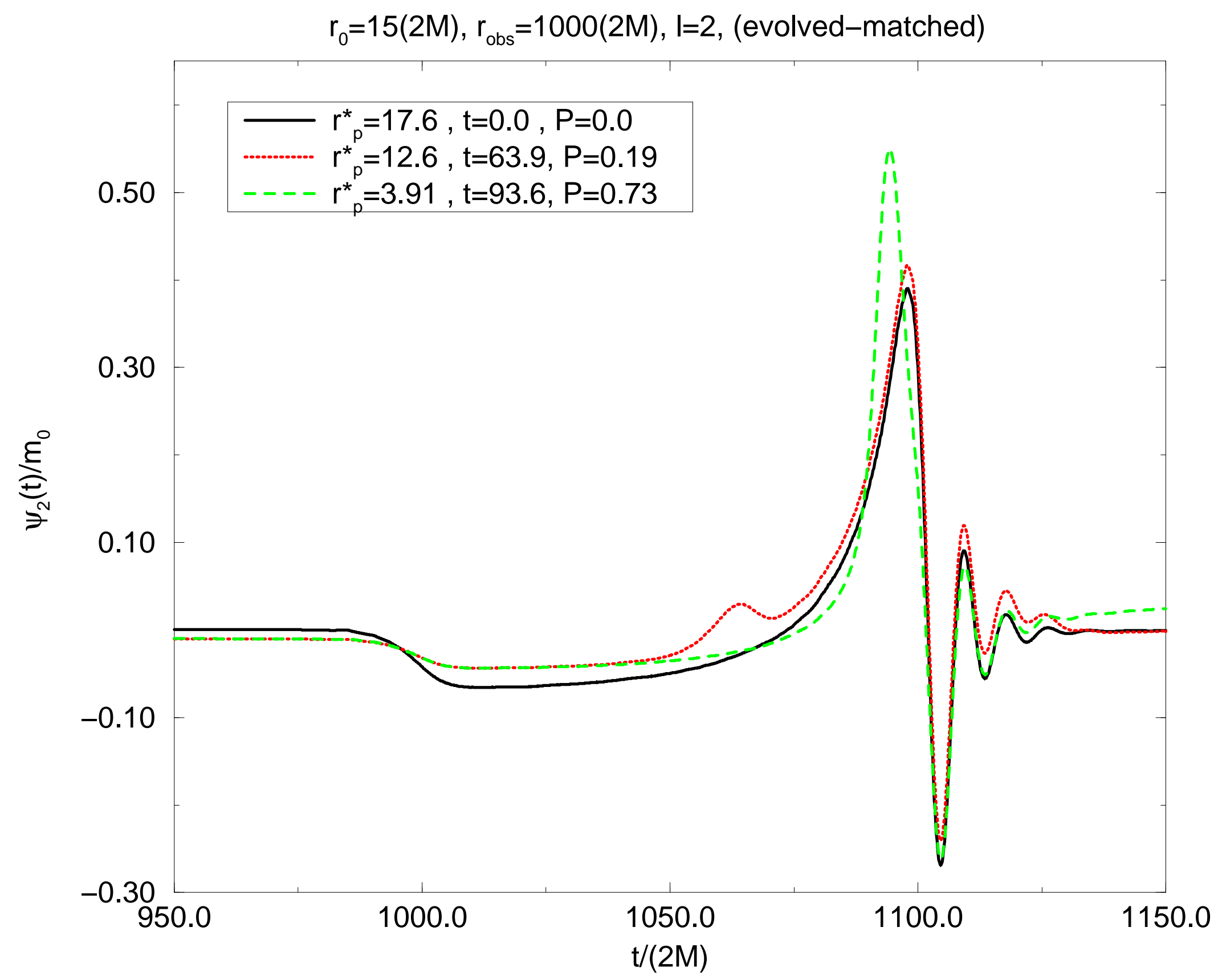




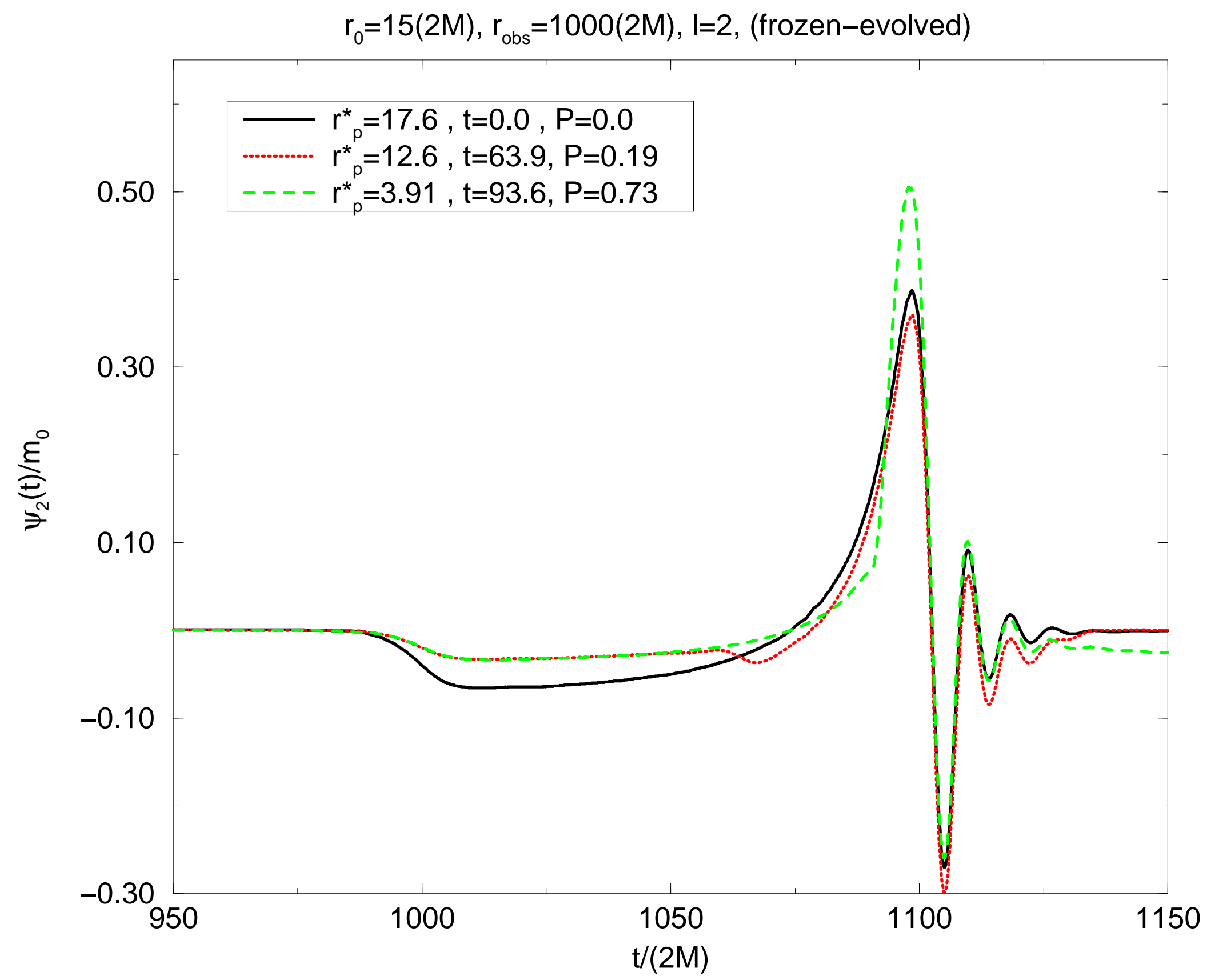




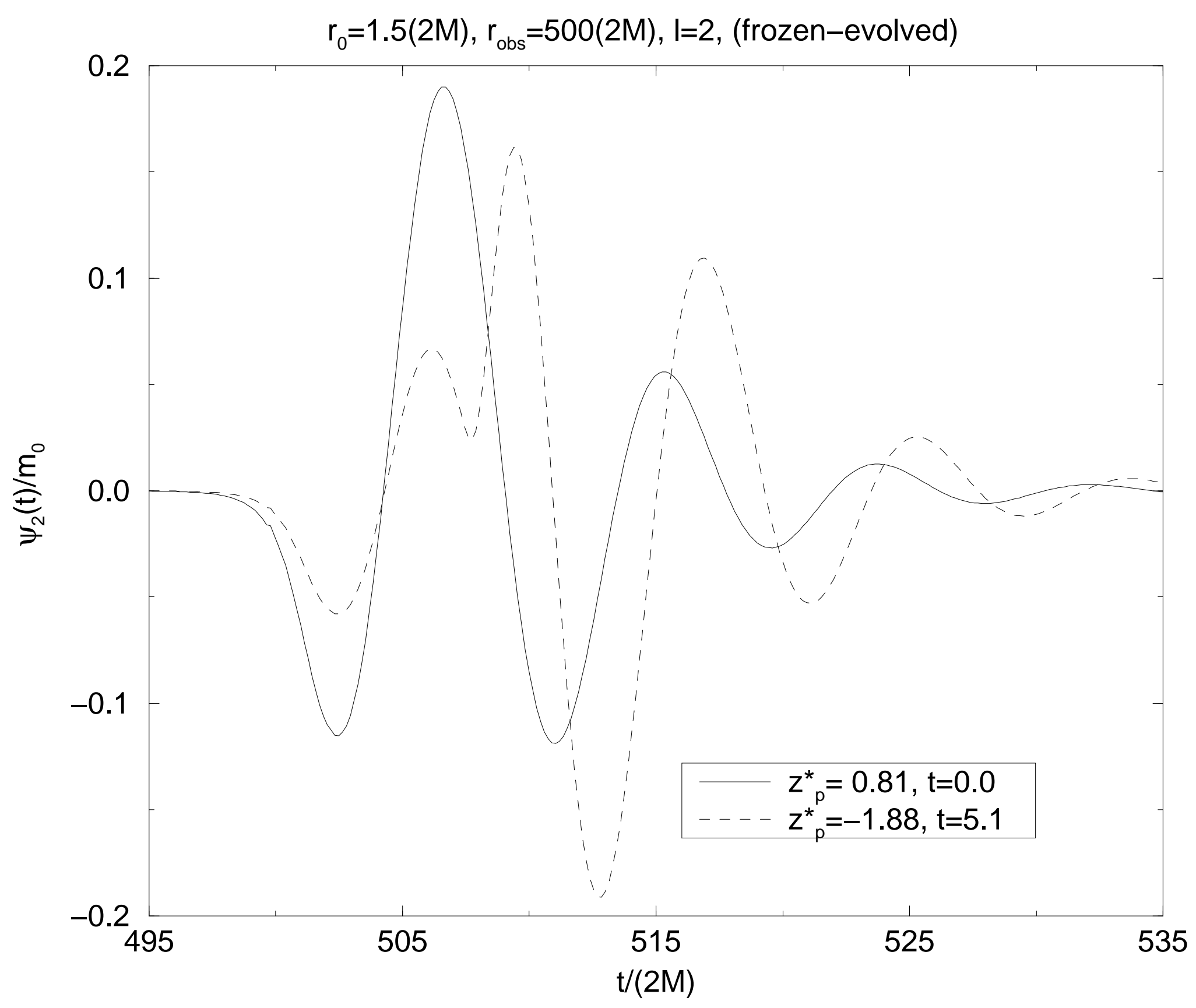




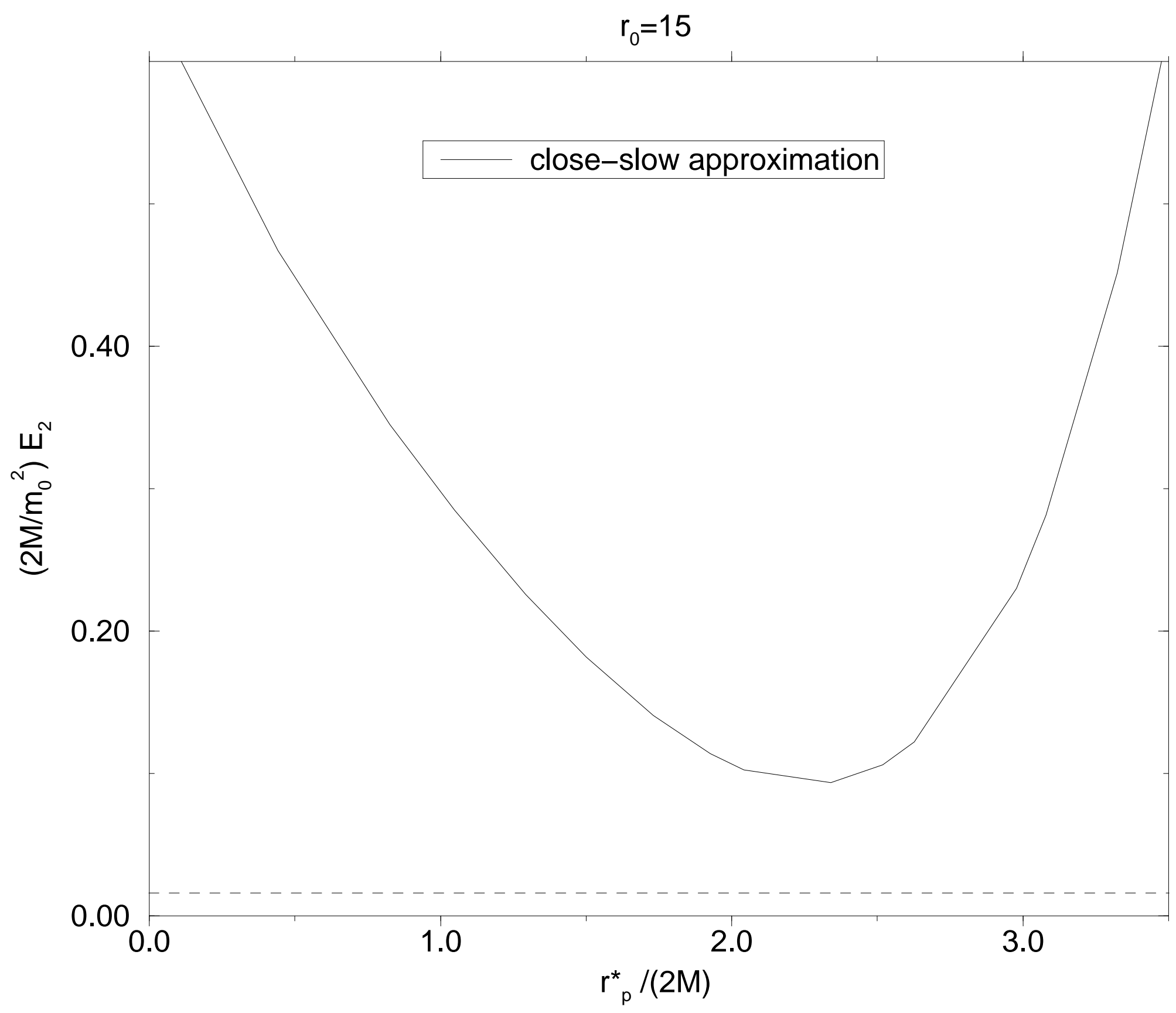

